TRANSACTIONS OF THE

AMERICAN MATHEMATICAL SOCIETY

Volume 358, Number 4, Pages 1619-1641

S 0002-9947(05)03750-5

Article electronically published on June 21, 2005

\title{
INNATELY TRANSITIVE SUBGROUPS OF WREATH PRODUCTS IN PRODUCT ACTION
}

\author{
ROBERT W. BADDELEY, CHERYL E. PRAEGER, AND CSABA SCHNEIDER
}

\begin{abstract}
A permutation group is innately transitive if it has a transitive minimal normal subgroup, which is referred to as a plinth. We study the class of finite, innately transitive permutation groups that can be embedded into wreath products in product action. This investigation is carried out by observing that such a wreath product preserves a natural Cartesian decomposition of the underlying set. Previously we classified the possible embeddings in the case where the plinth is simple. Here we extend that classification and identify several different types of Cartesian decompositions that can be preserved by an innately transitive group with a non-abelian plinth. These different types of decompositions lead to different types of embeddings of the acting group into wreath products in product action. We also obtain a full characterisation of embeddings of innately transitive groups with diagonal type into such wreath products.
\end{abstract}

\section{INTRODUCTION}

This paper forms an important part of our program to describe innately transitive subgroups of wreath products in product action (see Section 2 for the definition of wreath products and their product actions). A permutation group is innately transitive if it has a transitive minimal normal subgroup; and such a subgroup is called a plinth. A permutation group is said to be quasiprimitive if all its minimal normal subgroups are transitive. There are various characterisations of innately transitive and quasiprimitive groups: in BamP04 5 principal types of finite innately transitive groups were identified, and BadP03 listed 8 types of finite quasiprimitive permutation groups based on the classification obtained by Pra93. We use the types of [BamP04] and [BadP03] in this paper.

In an earlier paper [BPS04] we described those finite, innately transitive groups with a simple plinth that can be embedded into a wreath product in product action. The main results of this paper extend that classification to embeddings of innately transitive subgroups with a non-abelian plinth in such wreath products. Let $H$ be a quasiprimitive, almost simple permutation group acting on a set $\Gamma$. That is, $H$ has a unique minimal normal subgroup $U$, and $U$ is a non-abelian simple group. Set

Received by the editors December 18, 2003 and, in revised form, May 28, 2004.

2000 Mathematics Subject Classification. Primary 20B05, 20B15, 20B25, 20 B35.

Key words and phrases. Innately transitive groups, plinth, characteristically simple groups, Cartesian decompositions, Cartesian systems.

The authors acknowledge the support of an Australian Research Council grant. The third author was employed by The University of Western Australia as an ARC Research Associate while the research presented in this paper was carried out. We are very grateful to Laci Kovács for explaining the origins of some of the ideas that appear in this article. 
TABle 1. Table for Theorem 1.1

\begin{tabular}{|c|c|c|}
\hline & $T$ & $U$ \\
\hline \hline 1 & $\mathrm{~A}_{6}$ & $\mathrm{~A}_{6}$ \\
\hline 2 & $\mathrm{M}_{12}$ & $\mathrm{M}_{12}$ \\
\hline 3 & $\mathrm{M}_{12}$ & $\mathrm{~A}_{12}$ \\
\hline 4 & $\mathrm{P} \Omega_{8}^{+}(q)$ & $\mathrm{P} \Omega_{8}^{+}(q)$ \\
\hline 5 & $\mathrm{P} \Omega_{8}^{+}(q)$ & $\mathrm{A}_{n}$ where $n=\left|\mathrm{P} \Omega_{8}^{+}(q): \Omega_{7}(q)\right|$ \\
\hline 6 & $\mathrm{P} \Omega_{8}^{+}(2)$ & $\mathrm{Sp}_{8}(2)$ \\
\hline 7 & $\mathrm{Sp}_{4}\left(2^{a}\right), a \geqslant 2$ & $\mathrm{Sp}_{4 b}\left(2^{a / b}\right)$ where $b \mid a$ \\
\hline 8 & $\mathrm{Sp}_{4}\left(2^{a}\right), a \geqslant 2$ & $\mathrm{~A}_{n}$ where $n=\left|\mathrm{Sp}_{4}\left(2^{a}\right): \mathrm{Sp}_{2}\left(2^{2 a}\right) \cdot 2\right|$ \\
\hline
\end{tabular}

$W=H \mathrm{wr} \mathrm{S}_{\ell}$ for some $\ell \geqslant 2$, and consider $W$ as a permutation group in product action acting on $\Gamma^{\ell}$. Let $N=U_{1} \times \cdots \times U_{\ell}$ be the unique minimal normal subgroup of $W$; note that $N \cong U^{\ell}$. Assume that $G$ is an innately transitive subgroup of $W$ with a non-abelian plinth $M=T_{1} \times \cdots \times T_{k}$, where $T_{1}, \ldots, T_{k}$ are finite, non-abelian simple groups all isomorphic to a group $T$.

The following two theorems are the main results of this paper.

Theorem 1.1. If $G, W, M$, and $N$ are as above, then $M \leqslant N$. Further, if $G$ projects onto a transitive subgroup of $\mathrm{S}_{\ell}$, then exactly one of the following holds.

(a) $k=\ell$; the $T_{i}$ and the $U_{i}$ can be indexed so that $T_{1} \leqslant U_{1}, \ldots, T_{k} \leqslant U_{k}$.

(b) $\ell=2 k ; T$ and $U$ are as in Table 1; the $T_{i}$ and the $U_{i}$ can be indexed so that $T_{1} \leqslant U_{1} \times U_{2}, T_{2} \leqslant U_{3} \times U_{4}, \ldots, T_{k} \leqslant U_{2 k-1} \times U_{2 k}$.

(c) Neither of the cases (a)-(b) holds and $U=$ Alt $\Gamma$.

An even stronger result can be obtained if $G$ has diagonal type, which is defined as follows. Let $\sigma_{i}: M \rightarrow T_{i}$ be the natural projection map, for $i \in\{1, \ldots, k\}$. An innately transitive group $G$ has diagonal type if $\sigma_{i}\left(M_{\omega}\right)=T_{i}$ for all $\omega \in \Gamma^{\ell}$ and $i \in\{1, \ldots, k\}$. It follows from Scott's Lemma 4.1 that in this case a point stabiliser $M_{\omega}$ is isomorphic to $T^{s}$ for some $s \geqslant 1$. If $s=1$, then we say that $G$ has simple diagonal type, otherwise $G$ has compound diagonal type. It was shown in BamP04, Proposition 5.5] that an innately transitive group of diagonal type contains a unique minimal normal subgroup, and hence is quasiprimitive.

Theorem 1.2. If $W, G, N, M$ are as above and $G$ has diagonal type, then the following all hold.

(a) $G$ projects onto a transitive subgroup of $\mathrm{S}_{\ell}$ and $G$ is a quasiprimitive group of compound diagonal type;

(b) $U=$ Alt $\Gamma$;

(c) $M \leqslant N ; k=m \ell$ for some $m \geqslant 2$ and the $U_{i}$ and the $T_{i}$ can be indexed so that $T_{1} \times \cdots \times T_{m} \leqslant U_{1}, T_{m+1} \times \cdots \times T_{2 m} \leqslant U_{2}, \ldots, T_{(\ell-1) m+1} \times \cdots \times T_{\ell m} \leqslant$ $U_{\ell}$.

Theorem 1.2 implies the following important corollary, thus proving BadP03, Theorem 4.7(3)]. 
Corollary 1.3. An innately transitive permutation group with simple diagonal type can never be a subgroup of a wreath product $\mathrm{Sym}^{\mathrm{N}} \mathrm{wr} \mathrm{S}_{\ell}$ in product action with $|\Gamma| \geqslant 2$ and $\ell \geqslant 2$.

Note that cases (a) and (b) of Theorem 1.1 give detailed information about the embedding $G \leqslant W$, while case (c) contains a rich variety of examples which will be investigated further in Section 8 . The inclusions in case (a) are quite common. Take, for instance, finite simple permutation groups $T, U \leqslant$ Sym $\Gamma$ such that $T \leqslant U$. If $K$ is a transitive subgroup of $\mathrm{S}_{\ell}$, then clearly $T \mathrm{wr} K \leqslant U \mathrm{wr} \mathrm{S}_{\ell} \leqslant \operatorname{Sym} \Gamma^{\ell}$, and this inclusion is as in Theorem 1.1(a).

Embeddings belonging to Theorem 1.1(b) are not a great deal more mysterious, as illustrated by the following construction. By LPS87, the group Aut $\mathrm{A}_{6} \cong$ $\mathrm{P} \mathrm{L}_{2}(9)$, acting on a set of size 36 , can be embedded into $\mathrm{S}_{6} \mathrm{wr}_{2} \mathrm{~S}_{2}$ such that Aut $\mathrm{A}_{6}$ projects onto $\mathrm{S}_{2}$ via the natural projection map $\mathrm{S}_{6} \mathrm{wr}_{2} \rightarrow \mathrm{S}_{2}$. Hence if $K$ is a transitive subgroup of $S_{k}$ for some $k \geqslant 2$, then (Aut $A_{6}$ ) wr $K$ is a subgroup of $\left(\mathrm{S}_{6} \mathrm{wr} \mathrm{S}_{2}\right) \mathrm{wr} \mathrm{S}_{k}$, and, in turn, of $\mathrm{S}_{6} w r \mathrm{~S}_{2 k}$ in product action. It is easy to see that this inclusion (Aut $\mathrm{A}_{6}$ ) wr $K \leqslant \mathrm{~S}_{6} \mathrm{wr}_{2 k}$ is as in Theorem 1.1(b).

The easiest examples in case (c) are constructed by taking a simple permutation group $T \leqslant \operatorname{Sym} \Gamma$ and a transitive group $K \leqslant \mathrm{~S}_{m}$. Then $T$ wr $K$ is a subgroup of Sym $\Gamma$ wr $S_{m}$ in product action on $\Gamma^{m}$. Moreover if $L \leqslant S_{\ell}$ is a transitive permutation group, then clearly $(T \mathrm{wr} K) \mathrm{wr} L \leqslant \operatorname{Sym} \Gamma^{m} \mathrm{wr} \mathrm{S}_{\ell} \leqslant \operatorname{Sym} \Gamma^{m \ell}$. The inclusion $(T$ wr $K)$ wr $L \leqslant \operatorname{Sym}^{m}{ }^{m} \mathrm{wr}_{\ell}$ is as in Theorem 1.1(c). However, as we will see in Section 2, the most intriguing examples in case (c) cannot be obtained by this simple construction.

Note that, in Theorem 1.2 the fact that $G$ induces a transitive subgroup of $\mathrm{S}_{\ell}$ is a consequence of the hypotheses. An easy example of the inclusions described by Theorem 1.2 can be constructed as follows. Let $K$ be an innately transitive permutation group of simple diagonal type acting on $\Gamma$ and let $L$ be its unique minimal normal subgroup. Let $T$ denote the isomorphism type of a simple direct factor of $M$. Then for $\gamma \in \Gamma$, the point stabiliser $L_{\gamma}$ is isomorphic to $T$. If $Q$ is a transitive subgroup of $\mathrm{S}_{\ell}$, then $K$ wr $Q$ is an innately transitive group of compound diagonal type acting on $\Gamma^{\ell}$, and clearly $K \mathrm{wr} Q \leqslant \operatorname{Sym} \Gamma \mathrm{wr} \mathrm{S}_{\ell}$. This inclusion gives another example for Theorem 1.1(c).

In Section 2 we give three further examples of inclusions of innately transitive groups into wreath products in product action to illustrate the diversity and the beauty of the embeddings belonging to Theorem 1.1(c). Section 3 contains a brief account of Cartesian decompositions of sets and Cartesian systems of subgroups that were introduced in BPS04 and are fundamental to our approach.

The concept of Cartesian decompositions can be viewed as a straightforward generalisation of the concept of systems of product imprimitivity. Such systems were first defined by L. G. Kovács in his lecture at the Group Theory Conference at Oberwolfach in May 1987. The ideas presented in this Oberwolfach lecture played a crucial rôle in his paper Kov89b. In the terminology used by Kovács, a system of product imprimitivity for a group $G$ is a special type of Cartesian decomposition preserved by $G$, namely it is one on which $G$ acts transitively.

We draw attention to Theorem 5.1, which is of crucial importance in analysing the structure of Cartesian systems in $M$ in which the elements involve diagonal subgroups (which are called strips in our terminology introduced in Section 4) isomorphic to $T$, as in Example 2.3. This result may be compared with Scott's 
Lemma 4.1 on subdirect subgroups in characteristically simple groups that played a key rôle in proving the O'Nan-Scott Theorem for primitive groups.

Section 6]contains our major result, the 6-Class Theorem, about transitive Cartesian decompositions preserved by a finite innately transitive group. The examples in the Introduction and in Section 2 show that the classification in Theorem 1.1 can naturally be refined using the language of Cartesian systems. If $G \leqslant \mathrm{Sym} \Gamma \mathrm{wr} \mathrm{S}_{\ell}$ and $G$ induces a transitive subgroup of $\mathrm{S}_{\ell}$, then we say that the corresponding Cartesian decomposition is transitive. We find that for an innately transitive group $G$ with a non-abelian plinth, each of the transitive $G$-invariant Cartesian decompositions of the underlying set belongs to exactly one of the 6 classes identified by the 6-Class Theorem (Theorem 6.2). The class of a particular Cartesian decomposition can be determined from the abstract group structure of the subgroups in the corresponding Cartesian system. Cartesian decompositions in different classes lead to different types of embeddings of the group $G$ into wreath products in product action. This is strong evidence to support our belief that the theory of Cartesian decompositions and Cartesian systems is the appropriate framework for studying these embeddings.

If $G$ is an innately transitive permutation group with a non-abelian plinth, each transitive $G$-invariant Cartesian decomposition gives rise to a certain interesting combinatorial structure preserved by the group or its stabiliser subgroups. These combinatorial structures may be partitions (see Theorem 7.2), bipartite graphs, generalised graphs, depending on the class of the decomposition. In some classes it is also possible to restrict the abstract structure of the acting group (Theorem 6.3). In Section 7 we provide a characterisation of the Cartesian decompositions in two of the classes of the 6-Class Theorem (see Theorems 7.2 and 7.3 ). The investigation of the remaining 4 types can be found in [PS03 and [PS]. The main results of this article are proved in Section 8 .

It is also meaningful to study those embeddings of innately transitive permutation groups $G$ into wreath products $\operatorname{Sym} \Gamma \mathrm{wr} \mathrm{S}_{\ell}$ in product action in which $G$ induces an intransitive subgroup of $S_{\ell}$. This will be carried out in a separate paper.

Most of our results depend on the correctness of the finite simple group classification. For instance, a lot of information on the factorisations of simple and characteristically simple groups that depend on this classification are used throughout the paper. Theorem 5.1, which has central importance in the proof of our 6-Class Theorem and other theorems in Section 6, is a consequence of [BadP03, Lemma 2.2], whose proof uses the fact, verified by the finite simple group classification, that each automorphism of a non-abelian, finite simple group has a non-trivial fixed-point. The Schreier Conjecture about the outer automorphism group of a finite simple group is used in the proof of Lemma 8.1 .

In this paper we use the following notation. Permutations act on the right: if $\pi$ is a permutation and $\omega$ is a point, then the image of $\omega$ under $\pi$ is denoted $\omega \pi$. If $G$ is a group acting on a set $\Omega$, then $G^{\Omega}$ denotes the subgroup of Sym $\Omega$ induced by $G$.

\section{FURTHER EXAMPLES OF INCLUSIONS}

The examples of inclusions of innately transitive groups into wreath products in product action given in the Introduction are natural. In this section we give some 
further examples, whose existence, we believe, is really surprising. They indicate the richness of the theory described in this paper.

Let $\Gamma$ be a finite set, let $L \leqslant \operatorname{Sym} \Gamma$, let $\ell \geqslant 2$ be an integer, and let $H \leqslant \mathrm{~S}_{\ell}$. The wreath product $L$ wr $H$ is the semidirect product $L^{\ell} \rtimes H$, where, for $\left(x_{1}, \ldots, x_{\ell}\right) \in L^{\ell}$ and $\sigma \in \mathrm{S}_{\ell},\left(x_{1}, \ldots, x_{\ell}\right)^{\sigma^{-1}}=\left(x_{1 \sigma}, \ldots, x_{\ell \sigma}\right)$. The product action of $L \mathrm{wr} H$ is the action of $L$ wr $H$ on $\Gamma^{\ell}$ defined by

$$
\left(\gamma_{1}, \ldots, \gamma_{\ell}\right)\left(x_{1}, \ldots, x_{\ell}\right)=\left(\gamma_{1} x_{1}, \ldots, \gamma_{\ell} x_{\ell}\right) \quad \text { and } \quad\left(\gamma_{1}, \ldots, \gamma_{\ell}\right) \sigma^{-1}=\left(\gamma_{1 \sigma}, \ldots, \gamma_{\ell \sigma}\right)
$$

for all $\left(\gamma_{1}, \ldots, \gamma_{\ell}\right) \in \Gamma^{\ell}, x_{1}, \ldots, x_{\ell} \in L$, and $\sigma \in H$. The important properties of wreath products can be found in most textbooks on permutation group theory; see for instance [DM96.

Example 2.1. Let $T$ be a finite simple group and let $A, B$ be proper subgroups of $T$ such that $T=A B$. Set $K_{1}=A \times B$ and $K_{2}=B \times A$, and let $\Gamma_{1}$ and $\Gamma_{2}$ denote the right coset spaces $\left[T \times T: K_{1}\right]$ and $\left[T \times T: K_{2}\right]$, respectively. Note that $K_{1} K_{2}=T \times T$. Define $\pi \in \operatorname{Sym}\left(\Gamma_{1} \times \Gamma_{2}\right)$ as

$$
\left(K_{1}\left(t_{1}, t_{2}\right), K_{2}\left(t_{3}, t_{4}\right)\right) \pi=\left(K_{1}\left(t_{4}, t_{3}\right), K_{2}\left(t_{2}, t_{1}\right)\right)
$$

for all $t_{1}, t_{2}, t_{3}, t_{4} \in T$. It is routine to check that $\pi$ is an involution and that $\pi$ normalises Sym $\Gamma_{1} \times \operatorname{Sym} \Gamma_{2}$, interchanging Alt $\Gamma_{1}$ and Alt $\Gamma_{2}$. Let $\varrho_{1}$ and $\varrho_{2}$ be the permutation representations of $T \times T$ on $\Gamma_{1}$ and $\Gamma_{2}$, respectively, induced by right multiplication. Set

$$
M=\left\{\left(\varrho_{1}(t), \varrho_{2}(t)\right) \mid t \in T \times T\right\} .
$$

Clearly, $M$ is a subgroup of Sym $\Gamma_{1} \times \operatorname{Sym} \Gamma_{2}$, and the stabiliser in $M$ of the point $\left(K_{1}, K_{2}\right)$ is $M_{0}=\left\{\left(\varrho_{1}(t), \varrho_{2}(t)\right) \mid t \in K_{1} \cap K_{2}\right\}$. Since $K_{1} \cap K_{2}=(A \cap B) \times(A \cap B)$, it follows that $\left|M: M_{0}\right|=\left|T \times T: K_{1} \cap K_{2}\right|=|T|^{2} /|A \cap B|^{2}$. Further, since $T=A B$, we have, for $i=1,2$, that $\left|\Gamma_{i}\right|=\left|T \times T: K_{i}\right|=|T|^{2} /(|A| \cdot|B|)=$ $|T| /|A \cap B|$. Thus $\left|M: M_{0}\right|=\left|\Gamma_{1} \times \Gamma_{2}\right|$, and so $M$ is transitive on $\Gamma_{1} \times \Gamma_{2}$. Let $\alpha=\left(K_{1}\left(t_{1}, t_{2}\right), K_{2}\left(t_{3}, t_{4}\right)\right) \in \Gamma_{1} \times \Gamma_{2}$ and $y=\left(\varrho_{1}(t), \varrho_{2}(t)\right) \in M$ with $t=\left(x_{1}, x_{2}\right) \in$ $T \times T$; set $y^{\prime}=\left(\varrho_{1}\left(x_{2}, x_{1}\right), \varrho_{2}\left(x_{2}, x_{1}\right)\right)$. Then

$$
\begin{aligned}
\alpha y \pi=\left(K_{1}\left(t_{1} x_{1}, t_{2} x_{2}\right), K_{2}\left(t_{3} x_{1}, t_{4} x_{2}\right)\right) \pi= & \left(K_{1}\left(t_{4} x_{2}, t_{3} x_{1}\right), K_{2}\left(t_{2} x_{2}, t_{1} x_{1}\right)\right) \\
& =\left(K_{1}\left(t_{1}, t_{2}\right), K_{2}\left(t_{3}, t_{4}\right)\right) \pi y^{\prime}=\alpha \pi y^{\prime} .
\end{aligned}
$$

Since this is true for all $\alpha \in \Gamma_{1} \times \Gamma_{2}$, we have $y \pi=\pi y^{\prime}$, and so $y^{\pi}=y^{\prime}$ for all $y \in M$. As $y^{\prime} \in M, \pi$ normalises $M$. This simple argument also shows that

$$
\left(\varrho_{1}\left(x_{1}, x_{2}\right), \varrho_{2}\left(x_{1}, x_{2}\right)\right)^{\pi}=\left(\varrho_{1}\left(x_{2}, x_{1}\right), \varrho_{2}\left(x_{2}, x_{1}\right)\right) \text { for all } x_{1}, x_{2} \in T \text {. }
$$

Set

$$
T_{1}=\left\{\left(\varrho_{1}(t, 1), \varrho_{2}(t, 1)\right) \mid t \in T\right\} \quad \text { and } \quad T_{2}=\left\{\left(\varrho_{1}(1, t), \varrho_{2}(1, t)\right) \mid t \in T\right\} .
$$

Then $M$ can be written as the internal direct product $M=T_{1} \times T_{2}$. It also follows from (11) that $\pi$ interchanges $T_{1}$ and $T_{2}$. Let $W=\left(\operatorname{Sym} \Gamma_{1} \times \operatorname{Sym} \Gamma_{2}\right) \rtimes\langle\pi\rangle$ and $G=M \rtimes\langle\pi\rangle$. Then $G$ is a quasiprimitive group on $\Gamma_{1} \times \Gamma_{2}$ with unique minimal normal subgroup $M$, and $W$ is also quasiprimitive with minimal normal subgroup Alt $\Gamma_{1} \times$ Alt $\Gamma_{2}$. Moreover, as $\left|\Gamma_{1}\right|=\left|\Gamma_{2}\right|, W$ is permutationally isomorphic to the wreath product Sym $\Gamma_{1}$ wr $\mathrm{S}_{2}$ in product action. Therefore the inclusion $G \leqslant W$ is as in case (c) of Theorem 1.1. 
Example 2.2. Let $T$ be a finite simple group and let $A, B, C$ be subgroups of $T$ such that $T=A(B \cap C)=B(A \cap C)=C(A \cap B)$. Note that in this case we say that $\{A, B, C\}$ is a strong multiple factorisation of the finite simple group $T$, and the possibilities for $T, A, B$, and $C$ can be found in [BadP98, Table V]. Set $K_{1}=A \times B \times C, K_{2}=B \times C \times A$, and $K_{3}=C \times A \times B$; it is a routine calculation to check that

$$
K_{1}\left(K_{2} \cap K_{3}\right)=K_{2}\left(K_{1} \cap K_{3}\right)=K_{3}\left(K_{1} \cap K_{2}\right)=T^{3} .
$$

Let $\Gamma_{1}, \Gamma_{2}$, and $\Gamma_{3}$ denote the right coset spaces $\left[T^{3}: K_{1}\right],\left[T^{3}: K_{2}\right]$, and $\left[T^{3}: K_{3}\right]$, respectively, and define $\pi \in \operatorname{Sym}\left(\Gamma_{1} \times \Gamma_{2} \times \Gamma_{3}\right)$ as

$$
\begin{aligned}
& \left(K_{1}\left(t_{1}, t_{2}, t_{3}\right), K_{2}\left(t_{4}, t_{5}, t_{6}\right), K_{3}\left(t_{7}, t_{8}, t_{9}\right)\right) \pi \\
& \quad=\left(K_{1}\left(t_{8}, t_{9}, t_{7}\right), K_{2}\left(t_{2}, t_{3}, t_{1}\right), K_{3}\left(t_{5}, t_{6}, t_{4}\right)\right)
\end{aligned}
$$

for all $t_{1}, \ldots, t_{9} \in T$. It is routine to check that $\pi$ is a permutation of order 3 and that $\pi$ normalises Sym $\Gamma_{1} \times \operatorname{Sym} \Gamma_{2} \times \operatorname{Sym} \Gamma_{3}$, acting transitively on $\left\{\right.$ Alt $\Gamma_{1}$, Alt $\Gamma_{2}$, Alt $\left.\Gamma_{3}\right\}$ by conjugation. Let $\varrho_{1}, \varrho_{2}$, and $\varrho_{3}$ be the permutation representations of $T^{3}$ on $\Gamma_{1}, \Gamma_{2}$, and $\Gamma_{3}$, respectively, induced by right multiplication. Set

$$
M=\left\{\left(\varrho_{1}(t), \varrho_{2}(t), \varrho_{3}(t)\right) \mid t \in T^{3}\right\} .
$$

Clearly, $M$ is a subgroup of Sym $\Gamma_{1} \times \operatorname{Sym} \Gamma_{2} \times \operatorname{Sym} \Gamma_{3}$. Using (2), an argument similar to the one in Example 2.1 shows that $M$ is transitive on $\Gamma_{1} \times \Gamma_{2} \times \Gamma_{3}$. It is also easy to prove that $\pi$ normalises $M$. Set

$$
\begin{aligned}
& T_{1}=\left\{\left(\varrho_{1}(t, 1,1), \varrho_{2}(t, 1,1), \varrho_{3}(t, 1,1)\right) \mid t \in T\right\}, \\
& T_{2}=\left\{\left(\varrho_{1}(1, t, 1), \varrho_{2}(1, t, 1), \varrho_{3}(1, t, 1)\right) \mid t \in T\right\}, \text { and } \\
& T_{3}=\left\{\left(\varrho_{1}(1,1, t), \varrho_{2}(1,1, t), \varrho_{3}(1,1, t)\right) \mid t \in T\right\} .
\end{aligned}
$$

Then $M$ can be written as the internal direct product $M=T_{1} \times T_{2} \times T_{3}$, and $\pi$ acts transitively by conjugation on $T_{1}, T_{2}, T_{3}$. Let $W=\left(\operatorname{Sym} \Gamma_{1} \times \operatorname{Sym} \Gamma_{2} \times \operatorname{Sym} \Gamma_{3}\right) \rtimes\langle\pi\rangle$ and let $G=M \rtimes\langle\pi\rangle$. Then $G$ is a quasiprimitive group with minimal normal subgroup $M$, and $W$ is quasiprimitive with minimal normal subgroup Alt $\Gamma_{1} \times$ Alt $\Gamma_{2} \times$ Alt $\Gamma_{3}$. Moreover, as $\left|\Gamma_{1}\right|=\left|\Gamma_{2}\right|=\left|\Gamma_{3}\right|, W$ is permutationally isomorphic to the wreath product $\operatorname{Sym} \Gamma_{1}$ wr $\mathrm{A}_{3}$ in product action. Therefore $G$ can be viewed as a subgroup of Sym $\Gamma_{1} w r S_{3}$, and this inclusion is as in Theorem 1.1(c).

Example 2.3. Let $T$ be a finite simple group and let $A, B$ be proper isomorphic subgroups of $T$ such that $T=A B$. The possibilities for $T, A$, and $B$ can be found in [BPS04, Table 2]. We obtain from [BPS04, Lemma 5.2(ii)] that there exists a $\vartheta \in$ Aut $T$ that interchanges $A$ and $B$. Define the subgroups $K_{1}$ and $K_{2}$ of $T^{4}$ by

$$
K_{1}=\left\{\left(t_{1}, t_{2}, t_{3}, t_{4}\right) \mid t_{1} \in A, t_{2} \in B, t_{3}=t_{4}\right\}
$$

and

$$
K_{2}=\left\{\left(t_{1}, t_{2}, t_{3}, t_{4}\right) \mid t_{1}=t_{2}, t_{3} \in A, t_{4} \in B\right\} .
$$

One can check using PS02, Lemma 2.1] that $(A \times B)\{(t, t) \mid t \in T\}=T \times T$, and hence $K_{1} K_{2}=T^{4}$. Let $\Gamma_{1}$ and $\Gamma_{2}$ denote the right coset spaces $\left[T^{4}: K_{1}\right]$ and $\left[T^{4}: K_{2}\right]$, respectively. Define $\pi_{1} \in \operatorname{Sym} \Gamma_{1} \times \operatorname{Sym} \Gamma_{2}$ as follows:

$$
\begin{aligned}
& \left(K_{1}\left(t_{1}, t_{2}, t_{3}, t_{4}\right), K_{2}\left(t_{5}, t_{6}, t_{7}, t_{8}\right)\right) \pi_{1} \\
& \quad=\left(K_{1}\left(\vartheta\left(t_{2}\right), \vartheta\left(t_{1}\right), \vartheta\left(t_{4}\right), \vartheta\left(t_{3}\right)\right), K_{2}\left(\vartheta\left(t_{6}\right), \vartheta\left(t_{5}\right), \vartheta\left(t_{8}\right), \vartheta\left(t_{7}\right)\right)\right)
\end{aligned}
$$


for all $t_{1}, \ldots, t_{8} \in T$. Let $\pi_{2} \in \operatorname{Sym}\left(\Gamma_{1} \times \Gamma_{2}\right)$ be the permutation defined by

$$
\left(K_{1}\left(t_{1}, t_{2}, t_{3}, t_{4}\right), K_{2}\left(t_{5}, t_{6}, t_{7}, t_{8}\right)\right) \pi_{2}=\left(K_{1}\left(t_{7}, t_{8}, t_{5}, t_{6}\right), K_{2}\left(t_{3}, t_{4}, t_{1}, t_{2}\right)\right) .
$$

Set $\Pi=\left\langle\pi_{1}, \pi_{2}\right\rangle$. It is routine to check that $\pi_{2}$ is an involution that normalises Sym $\Gamma_{1} \times \operatorname{Sym} \Gamma_{2}$ swapping the subgroups Alt $\Gamma_{1}$ and Alt $\Gamma_{2}$. Let $\varrho_{1}$ and $\varrho_{2}$ be the permutation representations of $T^{4}$ on $\Gamma_{1}$ and $\Gamma_{2}$, respectively, induced by right multiplication. Set

$$
M=\left\{\left(\varrho_{1}(t), \varrho_{2}(t)\right) \mid t \in T^{4}\right\} .
$$

Then $M$ is a subgroup of Sym $\Gamma_{1} \times \operatorname{Sym} \Gamma_{2}$. Using the fact that $K_{1} K_{2}=T^{4}$, it is not hard to verify that $M$ is transitive on $\Gamma_{1} \times \Gamma_{2}$, and it also follows that $M$ is normalised by $\Pi$. Set

$$
\begin{aligned}
& T_{1}=\left\{\left(\varrho_{1}(t, 1,1,1), \varrho_{2}(t, 1,1,1)\right) \mid t \in T\right\}, \\
& T_{2}=\left\{\left(\varrho_{1}(1, t, 1,1), \varrho_{2}(1, t, 1,1)\right) \mid t \in T\right\}, \\
& T_{3}=\left\{\left(\varrho_{1}(1,1, t, 1), \varrho_{2}(1,1, t, 1)\right) \mid t \in T\right\}, \text { and } \\
& T_{4}=\left\{\left(\varrho_{1}(1,1,1, t), \varrho_{2}(1,1,1, t)\right) \mid t \in T\right\} .
\end{aligned}
$$

Then $M$ can be written as the internal direct product $M=T_{1} \times T_{2} \times T_{3} \times T_{4}$, and $\Pi$ acts transitively on $T_{1}, T_{2}, T_{3}, T_{4}$. Let $W=\left(\operatorname{Sym} \Gamma_{1} \times \operatorname{Sym} \Gamma_{2}\right) \rtimes\left\langle\pi_{2}\right\rangle$ and $G=M \Pi$. Then $G$ is a quasiprimitive group with unique minimal normal subgroup $M$. Moreover, as $\left|\Gamma_{1}\right|=\left|\Gamma_{2}\right|, W$ quasiprimitive and is permutationally isomorphic to the wreath product Sym $\Gamma_{1} \mathrm{wr} \mathrm{S}_{2}$ in product action. Therefore the inclusion $G \leqslant W$ is as in case (c) in Theorem 1.1.

Note that in all three of the above examples, the crucial fact that made $M$ a transitive group on the Cartesian product was that the subgroups $K_{i}$ formed a special factorisation of the characteristically simple group $T^{2}$ in Example 2.1, $T^{3}$ in Example 2.2. and $T^{4}$ in Example 2.3. Crucial properties of these subgroups $K_{i}$ are encapsulated in the definition of Cartesian systems in Section 3.

\section{Cartesian decompositions and Cartesian systems}

In a previous paper BPS04 we studied the general problem of describing the innately transitive subgroups of wreath products in product action. The corresponding problem for primitive groups was solved by Pra90 and Kov89a, but for quasiprimitive groups it was left open in BadP03. Related problems were also addressed in Bau97. If an innately transitive group $G$ with plinth $M$ is a subgroup of such a wreath product $W$, then the underlying set can be viewed as a Cartesian product of smaller sets. The actions of the groups $W$ and $G$ preserve this Cartesian product. We made these ideas more precise by introducing the concept of a Cartesian decomposition of a set.

A Cartesian decomposition of a set $\Omega$ is a set $\left\{\Gamma_{1}, \ldots, \Gamma_{\ell}\right\}$ of partitions of $\Omega$ such that

$$
\left|\gamma_{1} \cap \cdots \cap \gamma_{\ell}\right|=1 \text { for all } \gamma_{1} \in \Gamma_{1}, \ldots, \gamma_{\ell} \in \Gamma_{\ell} .
$$

This property implies that the map $\omega \mapsto\left(\gamma_{1}, \ldots, \gamma_{\ell}\right)$, where for $i=1, \ldots, \ell$ the block $\gamma_{i} \in \Gamma_{i}$ is chosen so that $\omega \in \gamma_{i}$, is a well-defined bijection between $\Omega$ and $\Gamma_{1} \times \cdots \times \Gamma_{\ell}$. Thus the set $\Omega$ can naturally be identified with the Cartesian product $\Gamma_{1} \times \cdots \times \Gamma_{\ell}$. This definition was first suggested by [Kov89b].

If $G$ is a permutation group acting on $\Omega$, then a Cartesian decomposition $\mathcal{E}$ of $\Omega$ is said to be $G$-invariant if the partitions in $\mathcal{E}$ are permuted by $G$. For a 
permutation group $G \leqslant \operatorname{Sym} \Omega$, the symbol $\operatorname{CD}(G)$ denotes the set of $G$-invariant Cartesian decompositions of $\Omega$. If $\mathcal{E} \in \mathrm{CD}(G)$ and $G$ acts on $\mathcal{E}$ transitively, then $\mathcal{E}$ is said to be a transitive $G$-invariant Cartesian decomposition. The set of transitive $G$-invariant Cartesian decompositions of $\Omega$ is denoted $\mathrm{CD}_{\operatorname{tr}}(G)$.

If $L \leqslant \operatorname{Sym} \Gamma$ and $H \leqslant \mathrm{~S}_{\ell}$ with some $\ell \geqslant 2$, then the wreath product $W=L$ wr $H$ at the beginning of Section 2 is considered as a permutation group acting in product action on the set $\Gamma^{\ell}$. There is a natural Cartesian decomposition of $\Gamma^{\ell}$ preserved by $W$; namely, take $\mathcal{E}=\left\{\Gamma_{1}, \ldots, \Gamma_{\ell}\right\}$, where

$$
\Gamma_{i}=\left\{\left\{\left(\gamma_{1}, \ldots, \gamma_{\ell}\right) \mid \gamma_{i}=\gamma\right\} \mid \gamma \in \Gamma\right\} \quad \text { for } \quad i=1, \ldots, \ell .
$$

The reader can easily check that $\mathcal{E}$ is a $W$-invariant Cartesian decomposition of $\Gamma^{\ell}$, and that the $W$-actions on the set $\mathcal{E}$ and on the set of natural coordinate subgroups of the base group $L^{\ell}$ of $W$ are equivalent. We study innately transitive subgroups of wreath products in product action, such as $W$, via the natural Cartesian decomposition of the underlying set corresponding to the product action of $W$.

Suppose that $G$ is an innately transitive subgroup of Sym $\Omega$ with plinth $M$, and that $\mathcal{E}$ is a $G$-invariant Cartesian decomposition of $\Omega$. We proved in BPS04, Proposition 2.1] that each $\Gamma_{i} \in \mathcal{E}$ is an $M$-invariant partition of $\Omega$. Choose an element $\omega$ of $\Omega$ and let $\gamma_{1} \in \Gamma_{1}, \ldots, \gamma_{\ell} \in \Gamma_{\ell}$ be such that $\{\omega\}=\gamma_{1} \cap \cdots \cap \gamma_{\ell}$; set $K_{i}=M_{\gamma_{i}}$. Then [BPS04, Lemmas 2.2 and 2.3] imply that the set $\mathcal{K}_{\omega}(\mathcal{E})=$ $\left\{K_{1}, \ldots, K_{\ell}\right\}$ is invariant under conjugation by $G_{\omega}$, and moreover

$$
\begin{aligned}
\bigcap_{i=1}^{\ell} K_{i} & =M_{\omega} \text { and } \\
K_{i}\left(\bigcap_{j \neq i} K_{j}\right) & =M \text { for all } i \in\{1, \ldots, \ell\} .
\end{aligned}
$$

For an arbitrary transitive permutation group $M$ on $\Omega$, and a point $\omega \in \Omega$, a set $\mathcal{K}=\left\{K_{1}, \ldots, K_{\ell}\right\}$ of proper subgroups of $M$ is said to be a Cartesian system of subgroups with respect to $\omega$ for $M$, if (3) and (4) hold. If $M$ is an abstract group, then a set $\left\{K_{1}, \ldots, K_{\ell}\right\}$ of proper subgroups satisfying (4) is said to be a Cartesian system.

The reader can easily see that the subgroups $K_{i}$ in Examples 2.1, 2.2, and 2.3 form, in each example, a Cartesian system for the characteristically simple group $M$. These examples show the importance of Cartesian systems for constructing inclusions of an innately transitive group $G$ into wreath products in product action. In fact, Cartesian systems provide an effective way of identifying the set of $G$ invariant Cartesian decompositions from information internal to $G$.

Theorem 3.1 (Theorem 1.4 and Lemma 2.3 of [BPS04]). Let $G \leqslant \operatorname{Sym} \Omega$ be an innately transitive permutation group with plinth $M$. For a fixed $\omega \in \Omega$ the correspondence $\mathcal{E} \mapsto \mathcal{K}_{\omega}(\mathcal{E})$ is a bijection between the set of $G$-invariant Cartesian decompositions of $\Omega$ and the set of $G_{\omega}$-invariant Cartesian systems of subgroups for $M$ with respect to $\omega$. Moreover the $G_{\omega}$-actions on $\mathcal{E}$ and on $\mathcal{K}_{\omega}(\mathcal{E})$ are equivalent.

Suppose that $G \leqslant \operatorname{Sym} \Omega$ is an innately transitive group with plinth $M$, and let $\omega \in \Omega$. Let $\mathcal{K}$ be a $G_{\omega}$-invariant Cartesian system of subgroups in $M$ with respect to $\omega$. Then the previous theorem implies that $\mathcal{K}=\mathcal{K}_{\omega}(\mathcal{E})$ for some $G$-invariant 
Cartesian decomposition $\mathcal{E}$ of $\Omega$. Indeed, it is easy to see that $\mathcal{E}$ consists of the $M$-invariant partitions $\left\{\left(\omega^{K}\right)^{m} \mid m \in M\right\}$, where $K$ runs through the elements of $\mathcal{K}$. This Cartesian decomposition is denoted $\mathcal{E}(\mathcal{K})$.

Using this theory we were able to describe in BPS04 those innately transitive subgroups of wreath products that have a simple plinth. This led to a classification of transitive simple and almost simple subgroups of wreath products in product action (see BPS04, Theorem 1.1]). Our aim in this paper is to extend that theory to achieve a more complete characterisation of innately transitive subgroups of wreath products in product action for innately transitive groups with a non-abelian plinth. As noted earlier, the case of abelian plinth was settled in Pra90.

\section{ON CHARACTERISTICALLY SIMPLE GROUPS}

Property (4) is of crucial importance for investigating Cartesian systems in characteristically simple groups. Hence it is very important for our research to study factorisations of such groups. In addition to the results in [PS02], we use the ones listed in this section.

Suppose that $T$ is a finite, non-abelian simple group and $\mathcal{L}$ is a set of proper subgroups in $T$ such that $|\mathcal{L}| \geqslant 3$ and $A(B \cap C)=T$ whenever $A, B$, and $C$ are pairwise distinct elements of $\mathcal{L}$. Then the set $\mathcal{L}$ is said to be a strong multiple factorisation of $T$. Strong multiple factorisations of finite simple groups were classified in [BadP98, Table V]. It was proved that not all finite simple groups admit a strong multiple factorisation, and each such factorisation contains exactly three pairwise non-isomorphic subgroups.

Suppose that $M=T_{1} \times \cdots \times T_{k}$, where the $T_{i}$ are non-abelian, finite simple groups. For $I \subseteq\left\{T_{1}, \ldots, T_{k}\right\}$ the function $\sigma_{I}: M \rightarrow \prod_{T_{i} \in I} T_{i}$ is the natural projection map. We also write $\sigma_{i}$ for $\sigma_{\left\{T_{i}\right\}}$. A subgroup $X$ of $M$ is said to be a strip if for each $i=1, \ldots, k$ either $\sigma_{i}(X)=1$ or $\sigma_{i}(X) \cong X$. The set of $T_{i}$ such that $\sigma_{i}(X) \neq 1$ is called the support of $X$ and is denoted Supp $X$. If $T_{m} \in$ Supp $X$, then we also say that $X$ covers $T_{m}$. Two strips $X_{1}$ and $X_{2}$ are disjoint if $\operatorname{Supp} X_{1} \cap \operatorname{Supp} X_{2}=\emptyset$. A strip $X$ is said to be full if $\sigma_{i}(X)=T_{i}$ for all $T_{i} \in \operatorname{Supp} X$, and $X$ is called non-trivial if $|\operatorname{Supp} X| \geqslant 2$. A subgroup $K$ of $M$ is said to be subdirect if $\sigma_{i}(K)=T_{i}$ for all $i$.

We recall a well-known lemma on finite simple groups which can be found in $\mathrm{Sco} 80$.

Lemma 4.1. Let $M$ be a direct product of finitely many non-abelian, finite simple groups and let $H$ be a subdirect subgroup of $M$. Then $H$ is the direct product of pairwise disjoint full strips of $M$.

The following result gives a generalisation of Scott's Lemma. Let $M=T_{1} \times$ $\cdots \times T_{k}$ be a characteristically simple group where $T_{1}, \ldots, T_{k}$ are the simple normal subgroups of $M$. If $K$ is a subgroup of $M$ and $X$ is a non-trivial strip in $M$ such that $K=X \times \sigma_{\left\{T_{1}, \ldots, T_{k}\right\} \backslash \operatorname{Supp} X}(K)$, then we say that $X$ is involved in $K$.

Lemma 4.2. Let $M$ be a direct product $T_{1} \times \cdots \times T_{k}$, where the $T_{i}$ are non-abelian, pairwise isomorphic finite simple groups, and let $K$ be a subgroup of $M$ such that $\sigma_{m}(K)=T_{m}$ for some $m \leqslant k$. Then there is a unique full strip $X$ of $M$ covering $T_{m}$ such that $X$ is involved in $K$. 
Proof. Let $X, Y$ be normal subgroups of $K$ which are minimal by inclusion subject to satisfying $\sigma_{m}(X)=\sigma_{m}(Y)=T_{m}$; such subgroups exist as $\sigma_{m}(K)=T_{m}$. Then

$$
\sigma_{m}(X \cap Y) \geqslant \sigma_{m}([X, Y])=\left[\sigma_{m}(X), \sigma_{m}(Y)\right]=\left[T_{m}, T_{m}\right]=T_{m}
$$

and by minimality we have $X=X \cap Y=Y$. Thus $K$ has a unique normal subgroup, $X$ say, which is minimal by inclusion subject to satisfying $\sigma_{m}(X)=T_{m}$.

We show that $X \cong T_{1}$. For $t \neq m$ consider $\sigma_{t}(X)$; suppose that this is non-trivial whence $X \cap \operatorname{ker} \sigma_{t}$ is a proper subgroup of $X$. As $X \cap \operatorname{ker} \sigma_{t}$ is normal in $X$ we deduce that $\sigma_{m}\left(X \cap \operatorname{ker} \sigma_{t}\right)$ is a normal subgroup of $T_{m}$ and is not equal to $T_{m}$ by the minimality of $X$. Thus $\sigma_{m}\left(X \cap \operatorname{ker} \sigma_{t}\right)$ is trivial, and so $X \cap \operatorname{ker} \sigma_{t} \leqslant X \cap \operatorname{ker} \sigma_{m}$. The latter subgroup has index $\left|T_{m}\right|$ in $X$, while the former has index dividing $\left|T_{t}\right|=\left|T_{m}\right|$. This forces $X \cap \operatorname{ker} \sigma_{m}=X \cap \operatorname{ker} \sigma_{t}$ and $\sigma_{t}(X)=T_{t}$. So for each $t=1, \ldots, k$ we have either $\sigma_{t}(X)=1$, or $\sigma_{t}(X)=T_{t}$ and $X \cap \operatorname{ker} \sigma_{t}=X \cap \operatorname{ker} \sigma_{m}$. Hence $\sigma_{t}\left(X \cap \operatorname{ker} \sigma_{m}\right)=1$ for all $t$, whence $X \cap \operatorname{ker} \sigma_{m}$ is trivial and $X \cong T_{m} \cong T_{1}$.

Thus $X$ is a full strip which covers $T_{m}$. As $X$ is self-normalising in $\prod_{T_{t} \in \operatorname{Supp} X} T_{t}$ (see [BamP04, Lemma 4.6]), we see that $\sigma_{\text {Supp } X}(K)=X$ whence $K=X \times$ $\sigma_{\left\{T_{1}, \ldots, T_{k}\right\} \backslash \operatorname{Supp} X}(K)$. Hence $X$ is involved in $K$.

The next lemma implies that the factorisation of a characteristically simple group as a product of a full strip and a proper subgroup is possible only under restricted conditions. This result is crucial for the proof of Theorem 6.1.

Lemma 4.3. Suppose that $M=T_{1} \times \cdots \times T_{k}$ is a non-abelian characteristically simple group, $\alpha_{i}: T_{1} \rightarrow T_{i}$ is an isomorphism for $i=2, \ldots, k$, and $K$ is a subgroup of $M$ such that $\sigma_{i}(K) \neq T_{i}$ for $i=1, \ldots, k$. If

$$
\left\{\left(t, \alpha_{2}(t), \ldots, \alpha_{k}(t)\right) \mid t \in T_{1}\right\} K=M,
$$

then $k \leqslant 3$. Moreover, if $k=3$, then $\left\{\sigma_{1}(K), \alpha_{2}^{-1}\left(\sigma_{2}(K)\right), \alpha_{3}^{-1}\left(\sigma_{3}(K)\right)\right\}$ is a strong multiple factorisation of $T_{1}$.

Proof. For each $t \in T_{1}$ there is some $x \in T_{1}$, and $\left(a_{1}, \ldots, a_{k}\right) \in K$ such that

$$
(t, 1, \ldots, 1)=\left(x, \alpha_{2}(x), \ldots, \alpha_{k}(x)\right)\left(a_{1}, \ldots, a_{k}\right) .
$$

Then $t=x a_{1}$ and $x=\alpha_{i}^{-1}\left(a_{i}^{-1}\right)$ for all $i=2, \ldots, k$, and so $x \in \alpha_{2}^{-1}\left(\sigma_{2}(K)\right) \cap \cdots \cap$ $\alpha_{k}^{-1}\left(\sigma_{k}(K)\right)$. So

$$
T_{1}=\sigma_{1}(K)\left(\alpha_{2}^{-1}\left(\sigma_{2}(K)\right) \cap \cdots \cap \alpha_{k}^{-1}\left(\sigma_{k}(K)\right)\right),
$$

and we obtain similarly that

$$
\begin{aligned}
& T_{1}=\alpha_{2}^{-1}\left(\sigma_{2}(K)\right)\left(\sigma_{1}(K) \cap \alpha_{3}^{-1}\left(\sigma_{3}(K)\right) \cap \cdots \cap \alpha_{k}^{-1}\left(\sigma_{k}(K)\right)\right) \\
&=\cdots=\alpha_{k}^{-1}\left(\sigma_{k}(K)\right)\left(\sigma_{1}(K) \cap \alpha_{2}^{-1}\left(\sigma_{2}(K)\right) \cap \cdots \cap \alpha_{k-1}^{-1}\left(\sigma_{k-1}(K)\right)\right) .
\end{aligned}
$$

Since $\sigma_{i}(K)$ is a proper subgroup of $T_{i}$ for all $i$, we obtain that

$$
\left\{\sigma_{1}(K), \alpha_{2}^{-1}\left(\sigma_{2}(K)\right), \ldots, \alpha_{k}^{-1}\left(\sigma_{k}(K)\right)\right\}
$$

is a strong multiple factorisation of $T_{1}$ whenever $k \geqslant 3$. Then the results of Baddeley and Praeger [BadP98] imply that $k=3$.

The next lemma which can be found as BadP03, Lemma 2.2] states that finite characteristically simple groups cannot be written as a product of two subgroups each of which is a direct product of non-trivial strips. The proof of this result uses 
the fact that each automorphism of a non-abelian, finite simple group has a nontrivial fixed-point, and hence it depends on the finite simple group classification.

Lemma 4.4. Suppose that $M=T_{1} \times \cdots \times T_{k}$ is a direct product of isomorphic nonabelian, simple groups $T_{1}, \ldots, T_{k}$. Suppose that $A_{1}, \ldots, A_{m}$ are non-trivial pairwise disjoint strips in $M$, and so are $B_{1}, \ldots, B_{n}$. Then

$$
M \neq\left(A_{1} \times \cdots \times A_{m}\right)\left(B_{1} \times \cdots \times B_{n}\right) .
$$

\section{Strips in a Cartesian system}

A non-trivial strip $X$ is said to be involved in a Cartesian system $\mathcal{K}$ for a nonabelian, characteristically simple group if $X$ is involved in an element of $\mathcal{K}$, as defined before Lemma 4.2. Note that in this case Lemma 4.4 and (4) imply that $X$ is involved in a unique element of $\mathcal{K}$. The purpose of this section is to prove that two distinct strips involved in a Cartesian system must be disjoint. This result plays a key rôle in the proof of our 6-Class Theorem (see in particular the proof of Theorem 6.1(d)).

Theorem 5.1. Let $M=T_{1} \times \cdots \times T_{k}$ be a finite, non-abelian, characteristically simple group with simple normal subgroups $T_{1}, \ldots, T_{k}$, and let $G_{0}$ be a subgroup of Aut $M$ such that the natural action of $G_{0}$ on $T_{1}, \ldots, T_{k}$ is transitive. Suppose, in addition, that $\mathcal{K}=\left\{K_{1}, \ldots, K_{\ell}\right\}$ is a $G_{0}$-invariant Cartesian system of subgroups in the abstract group $M$. If $X_{1}$ and $X_{2}$ are distinct, non-trivial strips involved in $\mathcal{K}$, then $X_{1}$ and $X_{2}$ are disjoint.

Proof. By the definition given above, if $X_{1}, X_{2}$ are involved in the same $K_{i}$, then they are disjoint as strips. Thus we may assume that $X_{1}$ is involved in $K_{j_{1}}$ and $X_{2}$ is involved in $K_{j_{2}}$ where $j_{1} \neq j_{2}$. First we prove that $\left|\operatorname{Supp} X_{1} \cap \operatorname{Supp} X_{2}\right| \leqslant 1$. Suppose to the contrary that $T_{i_{1}}, T_{i_{2}} \in \operatorname{Supp} X_{1} \cap \operatorname{Supp} X_{2}$ with $i_{1} \neq i_{2}$. Then $\sigma_{\left\{T_{i_{1}}, T_{i_{2}}\right\}}\left(K_{j_{1}}\right)$ and $\sigma_{\left\{T_{i_{1}}, T_{i_{2}}\right\}}\left(K_{j_{2}}\right)$ are non-trivial strips, and so Lemma 4.4 implies that $\sigma_{\left\{T_{i_{1}}, T_{i_{2}}\right\}}\left(K_{j_{1}}\right) \sigma_{\left\{T_{i_{1}}, T_{i_{2}}\right\}}\left(K_{j_{2}}\right) \neq T_{i_{1}} \times T_{i_{2}}$. However $K_{j_{1}} K_{j_{2}}=M$ by (4), which is a contradiction. Thus $\left|\operatorname{Supp} X_{1} \cap \operatorname{Supp} X_{2}\right| \leqslant 1$.

Assume that $\operatorname{Supp} X_{1} \cap \operatorname{Supp} X_{2}=\left\{T_{t}\right\}$ for some $t \leqslant k$. Choose $g \in G_{0}$ such that $T_{t}^{g} \in \operatorname{Supp} X_{2} \backslash \operatorname{Supp} X_{1}$; such an element $g$ exists since $G_{0}$ is transitive on $\mathcal{T}=\left\{T_{1}, \ldots, T_{k}\right\}$ and $\operatorname{Supp} X_{2} \backslash \operatorname{Supp} X_{1}$ is non-empty. Now $G_{0}$ acts by conjugation on the set of strips involved in $\mathcal{K}$, and so both $X_{1}^{g}$ and $X_{2}^{g}$ are strips involved in $\mathcal{K}$. As $T_{t}^{g}$ is in both $\operatorname{Supp} X_{1}^{g}$ and $\operatorname{Supp} X_{2}^{g}$ but is not in $\operatorname{Supp} X_{1}$, we deduce that there exists a non-trivial strip $X_{3}$ in $\mathcal{K}$ distinct from $X_{1}, X_{2}$ such that $\operatorname{Supp} X_{3} \cap$ $\left(\operatorname{Supp} X_{2} \backslash \operatorname{Supp} X_{1}\right) \neq \emptyset$ (namely, we can take $X_{3}$ to be one of $X_{1}^{g}$ or $X_{2}^{g}$ as at least one of these is distinct from $X_{1}$ and $X_{2}$ ). Proceeding in this way we construct a sequence $X_{1}, X_{2}, \ldots$ of distinct, non-trivial strips in $\mathcal{K}$ such that $\operatorname{Supp} X_{d+1} \cap$ (Supp $\left.X_{d} \backslash \operatorname{Supp} X_{d-1}\right) \neq \emptyset$ for each $d \geqslant 2$. Let $X_{a}$ be the first member of the sequence with $a \geqslant 3$ and

$$
\operatorname{Supp} X_{a} \cap\left(\operatorname{Supp} X_{1} \cup \cdots \cup \operatorname{Supp} X_{a-2}\right) \neq \emptyset \text {. }
$$

By removing some initial segment of the sequence if necessary we may assume that the intersection $\operatorname{Supp} X_{a} \cap \operatorname{Supp} X_{1}$ is non-empty, while Supp $X_{a} \cap \operatorname{Supp} X_{d}=\emptyset$ if $2 \leqslant d \leqslant a-2$ for some $a \geqslant 3$.

By relabeling the $K_{s}$ we may assume that $X_{1}$ is involved in $K_{1}$. Let $1=i_{1}<$ $i_{2}<\cdots<i_{d}<a$ be such that among the $X_{i}$ the strips $X_{i_{1}}, \ldots, X_{i_{d}}$ are precisely the ones that are involved in $K_{1}$. Note that $X_{a}$ is not involved in $K_{1}$ since Supp $X_{a}$ 
and Supp $X_{1}$ are not disjoint. Also, $i_{j+1} \geqslant i_{j}+2$ for all $j=1, \ldots, d-1$ since $\operatorname{Supp} X_{i_{j}}$ and $\operatorname{Supp} X_{i_{j}+1}$ are not disjoint. We may also relabel the $T_{i}$ so that

$$
\left\{T_{1}\right\}=\operatorname{Supp} X_{a} \cap \operatorname{Supp} X_{1} \quad \text { and } \quad\left\{T_{2}\right\}=\operatorname{Supp} X_{1} \cap \operatorname{Supp} X_{2},
$$

and so that for $j=2, \ldots, d$,

$$
\left\{T_{2 j-1}\right\}=\operatorname{Supp} X_{i_{j}-1} \cap \operatorname{Supp} X_{i_{j}} \quad \text { and } \quad\left\{T_{2 j}\right\}=\operatorname{Supp} X_{i_{j}} \cap \operatorname{Supp} X_{i_{j}+1} .
$$

It follows from the definition of $a$ that $T_{1}, \ldots, T_{2 d}$ are pairwise distinct. Let $\sigma$ be the projection map $M \rightarrow T_{1} \times \cdots \times T_{2 d}$. The labeling of $T_{1}, \ldots, T_{2 d}$ and the minimality of $a$ have ensured that the following all hold:

(i) $\sigma\left(X_{i_{j}}\right)$ is a non-trivial strip of $T_{2 j-1} \times T_{2 j}$ for $j=1, \ldots, d$;

(ii) for $j=1, \ldots, d-1$ we have

$$
\begin{aligned}
& \sigma\left(X_{i_{j}+1} \cap \cdots \cap X_{i_{j+1}-1}\right) \\
& \quad= \begin{cases}1 & \text { if } i_{j+1} \geqslant i_{j}+3, \\
\text { a non-trivial strip covering } T_{2 j} \text { and } T_{2 j+1} & \text { if } i_{j+1}=i_{j}+2 ;\end{cases}
\end{aligned}
$$

(iii) moreover,

$$
\sigma\left(X_{i_{d}+1} \cap \cdots \cap X_{a}\right)= \begin{cases}1 & \text { if } a \geqslant i_{d}+2, \\ \text { a non-trivial strip covering } T_{2 d} \text { and } T_{1} & \text { if } a=i_{d}+1 .\end{cases}
$$

Recalling that $X_{i_{1}}, \ldots, X_{i_{d}}$ are precisely the strips from the sequence $X_{1}, \ldots, X_{a}$ that appear as direct factors of $K_{1}$, we have that

$$
\sigma\left(K_{1}\right)=\sigma\left(X_{i_{1}} \times \cdots \times X_{i_{d}}\right),
$$

and that for $\widehat{K}_{1}=K_{2} \cap \cdots \cap K_{\ell}$,

$$
\sigma\left(\widehat{K}_{1}\right) \leqslant \sigma\left(\left(X_{2} \cap \cdots \cap X_{i_{2}-1}\right) \times \cdots \times\left(X_{i_{d}+1} \cap \cdots \cap X_{a}\right)\right) .
$$

From (i) we deduce that $\sigma\left(K_{1}\right)$ is the direct product of strips of $T_{1} \times T_{2}, \ldots, T_{2 d-1} \times$ $T_{2 d}$, while from (ii) and (iii) we deduce that $\sigma\left(\widehat{K}_{1}\right)$ is contained in the direct product of non-trivial strips of $T_{2} \times T_{3}, \ldots, T_{2 d-2} \times T_{2 d-1}, T_{2 d} \times T_{1}$. Thus $\sigma\left(K_{1}\right)$ and $\sigma\left(\widehat{K}_{1}\right)$ are each contained in the direct product of disjoint non-trivial strips of $T_{1} \times \cdots \times T_{2 d}$. Lemma 4.4 implies that $\sigma\left(K_{1}\right) \sigma\left(\widehat{K}_{1}\right) \neq \sigma(M)$, which contradicts the fact implied by (4) that $K_{1} \widehat{K}_{1}=M$. Thus $\operatorname{Supp} X_{1} \cap \operatorname{Supp} X_{2}=\emptyset$, as required.

\section{Six Classes of Cartesian Decompositions}

A non-abelian plinth of an innately transitive group $G$ has the form $M=T_{1} \times$ $\cdots \times T_{k}$, where the $T_{i}$ are finite, non-abelian, simple groups. Let $\mathcal{E} \in \operatorname{CD}(G)$ and let $\mathcal{K}_{\omega}(\mathcal{E})$ be a corresponding Cartesian system $\left\{K_{1}, \ldots, K_{\ell}\right\}$ for $M$ with respect to some $\omega \in \Omega$. Then equation (4) implies that, for all $i \leqslant k$ and $j \leqslant \ell$,

$$
\sigma_{i}\left(K_{j}\right)\left(\bigcap_{j^{\prime} \neq j} \sigma_{i}\left(K_{j^{\prime}}\right)\right)=T_{i} .
$$

In particular this means that if $\sigma_{i}\left(K_{j}\right)$ is a proper subgroup of $T_{i}$, then $\sigma_{i}\left(K_{j^{\prime}}\right) \neq$ $\sigma_{i}\left(K_{j}\right)$ for all $j^{\prime} \in\{1, \ldots, \ell\} \backslash\{j\}$. It is thus important to understand the following sets of subgroups:

$$
\mathcal{F}_{i}(\mathcal{E}, M, \omega)=\left\{\sigma_{i}\left(K_{j}\right) \mid j=1, \ldots, \ell, \sigma_{i}\left(K_{j}\right) \neq T_{i}\right\} .
$$


Note that $\left|\mathcal{F}_{i}(\mathcal{E}, M, \omega)\right|$ is the number of indices $j$ such that $\sigma_{i}\left(K_{j}\right) \neq T_{i}$. The set $\mathcal{F}_{i}(\mathcal{E}, M, \omega)$ is independent of $i$ up to isomorphism, in the sense that if $i_{1}, i_{2} \in$ $\{1, \ldots, k\}$ and $g \in G_{\omega}$ are such that $T_{i_{1}}^{g}=T_{i_{2}}$, then $\mathcal{F}_{i_{1}}(\mathcal{E}, M, \omega)^{g}=\left\{L^{g} \mid L \in\right.$ $\left.\mathcal{F}_{i_{1}}(\mathcal{E}, M, \omega)\right\}=\mathcal{F}_{i_{2}}(\mathcal{E}, M, \omega)$. This argument also shows that the elements of $\mathcal{F}_{i_{1}}(\mathcal{E}, M, \omega)$ are actually $G_{\omega}$-conjugate to the elements of $\mathcal{F}_{i_{2}}(\mathcal{E}, M, \omega)$.

Recall that, for $G \leqslant \operatorname{Sym} \Omega, \mathrm{CD}_{\operatorname{tr}}(G)$ denotes the set of transitive $G$-invariant Cartesian decompositions of $\Omega$. The following theorem depends on the finite simple group classification, since the proof uses results from BadP98 on full factorisations and multiple factorisations of finite simple groups.

Theorem 6.1. Suppose that $G$ is an innately transitive permutation group with a non-abelian plinth $M=T_{1} \times \cdots \times T_{k}$, where $T_{1}, \ldots, T_{k}$ are pairwise isomorphic finite simple groups and $k \geqslant 1$. Let $\mathcal{E} \in \mathrm{CD}_{\mathrm{tr}}(G)$ with a corresponding Cartesian system $\mathcal{K}$ for $M$ with respect to $\omega \in \Omega$. For $i=1, \ldots, k$, let $\mathcal{F}_{i}=\mathcal{F}_{i}(\mathcal{E}, M, \omega)$ be defined as in (6). Then the following all hold.

(a) The number $\left|\mathcal{F}_{i}\right|$ is independent of $i$ and $\left|\mathcal{F}_{i}\right| \leqslant 3$. Further, if $\left|\mathcal{F}_{i}\right|=3$, then $\mathcal{F}_{i}$ is a strong multiple factorisation of $T_{i}$.

(b) Suppose that there is a non-trivial, full strip involved in $\mathcal{K}$. Then $k \geqslant 2$ and $\left|\mathcal{F}_{i}\right| \in\{0,1\}$. Moreover, if $\left|\mathcal{F}_{i}\right|=1$, then the $T_{i}$ admit a factorisation as a product of two proper, isomorphic subgroups.

(c) If $X$ is a non-trivial, full strip involved in $\mathcal{K}$ and $\left|\mathcal{F}_{i}\right|=1$, then $|\operatorname{Supp} X|=$ 2 .

(d) Set $\mathcal{P}=\{\operatorname{Supp} X \mid X$ is a non-trivial, full strip involved in $\mathcal{K}\}$. If $\mathcal{P} \neq \emptyset$, then $\mathcal{P}$ is a $G$-invariant partition of $\left\{T_{1}, \ldots, T_{k}\right\}$.

Proof. (a) Suppose that $i_{1}, i_{2} \in\{1, \ldots, k\}$ and $g \in G_{\omega}$ such that $T_{i_{1}}^{g}=T_{i_{2}}$. Then it was observed after equation (6) that $\mathcal{F}_{i_{1}}^{g}=\mathcal{F}_{i_{2}}$, and so $\left|\mathcal{F}_{i}\right|$ is independent of $i$. The definition of $\mathcal{F}_{i}$ and (5) imply that if $\left|\mathcal{F}_{i}\right| \geqslant 3$, then $\mathcal{F}_{i}$ is a strong multiple factorisation of $T_{i}$. Hence [BadP98, Table V] shows that $\left|\mathcal{F}_{i}\right| \leqslant 3$.

(b) A non-trivial strip has to cover at least two of the $T_{i}$, and so we must have $k \geqslant 2$. We argue by contradiction and assume that $\left|\mathcal{F}_{i}\right| \geqslant 2$. Suppose without loss of generality that $K_{1}$ involves a non-trivial full strip $X$ covering $T_{1}$ and $T_{2}$. Let $g \in G_{\omega}$ such that $T_{1}^{g}=T_{2}$. Then, by Theorem 5.1, $X^{g}=X$, and so $K_{1}^{g}=K_{1}$, and also $\widehat{K}_{1}^{g}=\widehat{K}_{1}$, where, as in the proof of Theorem [5.1 $\widehat{K}_{1}=K_{2} \cap \cdots \cap K_{\ell}$. This also implies that $\sigma_{1}\left(\widehat{K}_{1}\right)^{g}=\sigma_{2}\left(\widehat{K}_{1}\right)$; therefore $\sigma_{1}\left(\widehat{K}_{1}\right)$ and $\sigma_{2}\left(\widehat{K}_{1}\right)$ are isomorphic. Note that

$$
\sigma_{\{1,2\}}(X)=\left\{(t, \alpha(t)) \mid t \in T_{1}\right\}
$$

for some isomorphism $\alpha: T_{1} \rightarrow T_{2}$. As $\left|\mathcal{F}_{1}\right| \geqslant 2$, we obtain that $\sigma_{1}\left(K_{j_{1}}\right)<T_{1}$ and $\sigma_{1}\left(K_{j_{2}}\right)<T_{1}$ for distinct $j_{1}, j_{2} \in\{2, \ldots, \ell\}$, and, as noted above, $\sigma_{1}\left(K_{j_{1}}\right) \neq$ $\sigma_{1}\left(K_{j_{2}}\right)$. Thus $\sigma_{1}\left(\widehat{K}_{1}\right)<T_{1}$ and, as $\sigma_{1}\left(\widehat{K}_{1}\right) \cong \sigma_{2}\left(\widehat{K}_{1}\right)$, also $\sigma_{2}\left(\widehat{K}_{1}\right)<T_{2}$. Since $K_{1} \widehat{K}_{1}=M$, PS02, Lemma 2.1] implies that $\sigma_{1}\left(\widehat{K}_{1}\right)$ and $\alpha^{-1}\left(\sigma_{2}\left(\widehat{K}_{1}\right)\right)$ form a full factorisation of $T_{1}$ with isomorphic subgroups. Based on the classification of full factorisations of almost simple groups in BadP98, such factorisations were described in [BPS04, Lemma 5.2]. In particular, $\sigma_{1}\left(\widehat{K}_{1}\right)$ and $\alpha^{-1}\left(\sigma_{2}\left(\widehat{K}_{1}\right)\right)$ are both maximal subgroups of $T_{1}$. On the other hand, $\sigma_{1}\left(\widehat{K}_{1}\right) \leqslant \sigma_{1}\left(K_{j_{1}}\right) \cap \sigma_{1}\left(K_{j_{2}}\right)<$ $\sigma_{1}\left(K_{j_{1}}\right), \sigma_{1}\left(K_{j_{2}}\right)$, which is a contradiction. Therefore $\left|\mathcal{F}_{i}\right| \leqslant 1$.

(c) Suppose without loss of generality that $X$ is a non-trivial, full strip involved in $K_{1}$ covering $T_{1}, \ldots, T_{s}$. Let $i, j$ be distinct elements of $\{1, \ldots, s\}$ and let $g \in G_{\omega}$ 
such that $T_{i}^{g}=T_{j}$. Then $X^{g}$ is a strip involved in $\mathcal{K}$ such that $T_{j} \in \operatorname{Supp} X \cap$ Supp $X^{g}$. Therefore by Theorem [5.1, $X=X^{g}$, and so $K_{1}^{g}=K_{1}$, and also $\widehat{K}_{1}^{g}=$ $\widehat{K}_{1}$. Since $T_{i}^{g}=T_{j}$ it follows that $\sigma_{i}\left(\widehat{K}_{1}\right)^{g}=\sigma_{j}\left(\widehat{K}_{1}\right)$. Now, as $\left|\mathcal{F}_{i}\right|=1$, there exists $t$ such that $\sigma_{i}\left(K_{t}\right)<T_{i}$, and, as $\sigma_{i}\left(K_{1}\right)=T_{i}$, we have $t \neq 1$. Hence $\sigma_{i}\left(\widehat{K}_{1}\right)<T_{i}$. Also, $\sigma_{j}\left(\widehat{K}_{1}\right)=\sigma_{i}\left(\widehat{K}_{1}\right)^{g}<T_{i}^{g}=T_{j}$. Therefore $\sigma_{i}\left(\widehat{K}_{1}\right)<T_{i}$ holds for all $i \in\{1, \ldots, s\}$. Thus the factorisation $\sigma_{\operatorname{Supp} X}(M)=\sigma_{\operatorname{Supp} X}\left(K_{1}\right) \sigma_{\operatorname{Supp} X}\left(\widehat{K}_{1}\right)$ is as in Lemma 4.3, and so $s \leqslant 3$, and moreover if $s=3$, then the projections $\sigma_{1}\left(\widehat{K}_{1}\right), \sigma_{2}\left(\widehat{K}_{1}\right)$, and $\sigma_{3}\left(\widehat{K}_{1}\right)$ are isomorphic to the subgroups in a strong multiple factorisation of $T_{1}$. On the other hand, the subgroups in such a strong multiple factorisation are pairwise non-isomorphic (see [BadP98, Table V]), a contradiction. Hence we obtain $s=2$.

(d) Let $\mathcal{P}=\{\operatorname{Supp} X \mid X$ is a non-trivial, full strip involved in $\mathcal{K}\}$. By Theorem 5.1, either $\mathcal{P}=\emptyset$ or $\mathcal{P}$ is a partition of $\left\{T_{1}, \ldots, T_{k}\right\}$. Moreover, if $\operatorname{Supp} X \in \mathcal{P}$ and $g \in G_{\omega}$, then there exists $K \in \mathcal{K}$ such that $X$ is involved in $K$. Therefore $K^{g} \in \mathcal{K}$ and $X^{g}$ is involved in $K^{g}$. Thus $X^{g}$ is involved in $\mathcal{K}$, and so $(\operatorname{Supp} X)^{g}=\operatorname{Supp}\left(X^{g}\right) \in \mathcal{P}$. Hence $\mathcal{P}$ is $G_{\omega}$-invariant. Since $M$ acts trivially on $\left\{T_{1}, \ldots, T_{k}\right\}$ by conjugation and $G=M G_{\omega}$, we have that $\mathcal{P}$ is a $G$-invariant partition of $\left\{T_{1}, \ldots, T_{k}\right\}$.

If $G$ is a finite, innately transitive group with a non-abelian plinth $M$, then the set $\mathrm{CD}_{\mathrm{tr}}(G)$ is further subdivided according to the structure of the subgroups in the corresponding Cartesian systems for $M$ as follows. For $\mathcal{E} \in \mathrm{CD}_{\operatorname{tr}}(G)$ and $\omega \in \Omega$ the sets $\mathcal{F}_{i}=\mathcal{F}_{i}(\mathcal{E}, M, \omega)$ are as defined in ([6).

$$
\begin{aligned}
\mathrm{CD}_{\mathrm{S}}(G) & =\left\{\mathcal{E} \in \mathrm{CD}_{\mathrm{tr}}(G) \mid \text { the elements of } \mathcal{K}_{\omega}(\mathcal{E}) \text { are subdirect subgroups in } M\right\} ; \\
\mathrm{CD}_{1}(G) & =\left\{\mathcal{E} \in \mathrm{CD}_{\mathrm{tr}}(G)|| \mathcal{F}_{i} \mid=1 \text { and } \mathcal{K}_{\omega}(\mathcal{E}) \text { involves no non-trivial, full strip }\right\} ; \\
\mathrm{CD}_{1 \mathrm{~S}}(G) & =\left\{\mathcal{E} \in \mathrm{CD}_{\operatorname{tr}}(G)|| \mathcal{F}_{i} \mid=1 \text { and } \mathcal{K}_{\omega}(\mathcal{E}) \text { involves non-trivial, full strips }\right\} ; \\
\mathrm{CD}_{2}(G) & =\left\{\mathcal{E} \in \mathrm{CD}_{\operatorname{tr}}(G)|| \mathcal{F}_{i} \mid=2 \text { and the } \mathcal{F}_{i} \text { contain two } G_{\omega} \text {-conjugate subgroups }\right\} ; \\
\mathrm{CD}_{2 \varkappa}(G) & =\left\{\mathcal{E} \in \mathrm{CD}_{\operatorname{tr}}(G)|| \mathcal{F}_{i} \mid=2 \text { and the subgroups in } \mathcal{F}_{i} \text { are not } G_{\omega} \text {-conjugate }\right\} ; \\
\mathrm{CD}_{3}(G) & =\left\{\mathcal{E} \in \mathrm{CD}_{\operatorname{tr}}(G)|| \mathcal{F}_{i} \mid=3\right\} .
\end{aligned}
$$

In order to prove that the above classes of Cartesian decompositions are well defined, we need to show that the properties used in these definitions are independent of the choice of the plinth $M$, the Cartesian system $\mathcal{K}_{\omega}(\mathcal{E})$, and the index $i$.

Theorem 6.2 (6-class Theorem). If $G$ is a finite, innately transitive permutation group with a non-abelian plinth $M$, then the classes $\mathrm{CD}_{1}(G), \mathrm{CD}_{\mathrm{S}}(G), \mathrm{CD}_{1 \mathrm{~S}}(G)$, $\mathrm{CD}_{2 \sim}(G), \mathrm{CD}_{2 \nsim}(G)$, and $\mathrm{CD}_{3}(G)$ are well-defined subsets of $\mathrm{CD}_{\mathrm{tr}}(G)$, and they form a partition of $\mathrm{CD}_{\mathrm{tr}}(G)$. Moreover, if $M$ is simple, then $\mathrm{CD}_{\mathrm{tr}}(G)=\mathrm{CD}_{2 \sim}(G)$.

Proof. First we prove that the above classes are well defined. Suppose that $\mathcal{E} \in$ $\mathrm{CD}_{\operatorname{tr}}(G)$, and let $\mathcal{E}=\left\{\Gamma_{1}, \ldots, \Gamma_{\ell}\right\}$. We need to show that the class of $\mathcal{E}$ does not depend on the choice of the plinth $M$, the point $\omega \in \Omega$, or the index $i \in\{1, \ldots, k\}$. First we verify that, given $M$, the class of $\mathcal{E}$ is independent of $\omega$ and $i$. Let $\omega_{1}, \omega_{2} \in$ $\Omega$, and let $\mathcal{K}_{1}=\mathcal{K}_{\omega_{1}}(\mathcal{E}), \mathcal{K}_{2}=\mathcal{K}_{\omega_{2}}(\mathcal{E})$ be the corresponding Cartesian systems for $M$. As $M$ is a transitive subgroup of $G$, there is some $m \in M$ such that $\omega_{1} m=\omega_{2}$ and [BPS04, Lemma 2.2] implies that $\mathcal{K}_{1}^{m}=\mathcal{K}_{2}$. Hence, an element $K$ of $\mathcal{K}_{1}$ involves a non-trivial, full strip $X$, if and only if the corresponding element $K^{m}$ of $\mathcal{K}_{2}$ involves the non-trivial full strip $X^{m}$. Thus the existence of a non-trivial strip involved in 
an element of a Cartesian system corresponding to $\mathcal{E}$ is independent of the choice of $\omega$. Moreover, $\sigma_{i}(K)<T_{i}$ holds for some $K \in \mathcal{K}_{1}$ if and only if $\sigma_{i}\left(K^{m}\right)<T_{i}$ holds. Thus the number $\left|\mathcal{F}_{i}(\mathcal{E}, M, \omega)\right|$ is independent of the choice $\omega$, and, by Theorem 6.1 (a), it is independent of $i$. It remains to prove that the definitions of the classes $\mathrm{CD}_{2 \sim}(G)$ and $\mathrm{CD}_{2 \chi}(G)$ are independent of $\omega$ and of $i$. The simple argument given after (6) shows that, for $j=1,2$, the elements of $\mathcal{F}_{i}\left(\mathcal{E}, M, \omega_{j}\right)$ are $G_{\omega_{j}}$-conjugate for some $i$ if and only if they are $G_{\omega_{j}}$-conjugate for all $i$. Suppose that the elements of $\mathcal{F}_{1}\left(\mathcal{E}, M, \omega_{1}\right)$ are $G_{\omega_{1}}$-conjugate. Let $\mathcal{F}_{1}\left(\mathcal{E}, M, \omega_{1}\right)=\{A, B\}$ and let $g \in G_{\omega_{1}}$ be such that $A^{g}=B$. Then $\mathcal{F}_{1}\left(\mathcal{E}, M, \omega_{1}\right)^{m}=\mathcal{F}_{1}\left(\mathcal{E}, M, \omega_{2}\right)$ and $A^{m g^{m}}=A^{g m}=B^{m}$, hence the elements $A^{m}$ and $B^{m}$ of $\mathcal{F}_{1}\left(\mathcal{E}, M, \omega_{2}\right)$ are conjugate under the element $g^{m}$. Further, $\omega_{2} g^{m}=\omega_{1} m g^{m}=\omega_{1} g m=\omega_{1} m=\omega_{2}$, and so $g^{m} \in G_{\omega_{2}}$. Thus the elements of $\mathcal{F}_{1}\left(\mathcal{E}, M, \omega_{2}\right)$ are conjugate under $G_{\omega_{2}}$. Hence the definitions of $\mathrm{CD}_{2 \sim}(G)$ and $\mathrm{CD}_{2 \varkappa}(G)$ are independent of the choice of $\omega$ and $i$.

Next we show that the class of $\mathcal{E}$ is independent of the plinth $M$. If $M$ is the unique transitive, minimal normal subgroup of $G$, then there is nothing to prove. If this is not the case, then $G$ has exactly two transitive, minimal normal subgroups $M$ and $\widehat{M}$, they are isomorphic, they both are regular on $\Omega$, and $\mathbb{C}_{G}(M)=\widehat{M}$, $\mathbb{C}_{G}(\widehat{M})=M$ also hold. Moreover, there is an involution $\alpha \in \mathbb{N}_{\text {Sym } \Omega}(G)$ that interchanges $M$ and $\widehat{M}$ (see BamP04, Lemma 5.1]). It follows from the proof of BamP04, Lemma 5.1] that this involution $\alpha$ lies in a point stabiliser. As $G$ is transitive on $\Omega$, we may assume without loss of generality that $\alpha$ is an element of the point stabiliser $\left(\mathbb{N}_{\text {Sym } \Omega}(G)\right)_{\omega}$. Let $\widehat{T}_{1}=T_{1}^{\alpha}, \ldots, \widehat{T}_{k}=T_{k}^{\alpha}$. Then $\widehat{M}=\widehat{T}_{1} \times \cdots \times \widehat{T}_{k}$. Let $\widehat{\sigma}_{i}: \widehat{M} \rightarrow \widehat{T}_{i}$ be the natural projection map, and define $\mathcal{F}_{1}(\mathcal{E}, M, \omega), \mathcal{F}_{1}(\mathcal{E}, \widehat{M}, \omega)$ as in (6). Let $\mathcal{K}$ and $\widehat{\mathcal{K}}$ be the Cartesian systems for $M$ and $\widehat{M}$, respectively, with respect to $\omega$. For $i=1, \ldots, \ell$, let $\gamma_{i} \in \Gamma_{i}$ such that $\omega \in \gamma_{i}$. As $\omega \alpha=\omega$, we obtain that $\widehat{M}_{\gamma_{i}}=\left(M_{\gamma_{i}}\right)^{\alpha}$, and so, by the definition of $\mathcal{K}$ and $\widehat{\mathcal{K}}$, we have $\widehat{\mathcal{K}}=\mathcal{K}^{\alpha}=\left\{K^{\alpha} \mid K \in \mathcal{K}\right\}$. Let $t_{1} \cdots t_{k} \in M$ with $t_{1} \in T_{1}, \ldots, t_{k} \in T_{k}$. Then $t_{1}^{\alpha} \cdots t_{k}^{\alpha} \in \widehat{M}$ with $t_{1}^{\alpha} \in \widehat{T}_{1}, \ldots, t_{k}^{\alpha} \in \widehat{T}_{k}$. Thus $\sigma_{i}\left(t_{1} \cdots t_{k}\right)^{\alpha}=\widehat{\sigma}_{i}\left(\left(t_{1} \cdots t_{k}\right)^{\alpha}\right)$ for all $i \in\{1, \ldots, k\}$. Therefore

$$
\widehat{\sigma}_{1}\left(K^{\alpha}\right)=\sigma_{1}(K)^{\alpha} \quad \text { for all } \quad K \in \mathcal{K}
$$

and so $\sigma_{1}(K) \neq T_{1}$ if and only if $\widehat{\sigma}_{1}\left(K^{\alpha}\right) \neq \widehat{T}_{1}$. Hence $\left|\mathcal{F}_{1}(\mathcal{E}, M, \omega)\right|=\left|\mathcal{F}_{1}(\mathcal{E}, \widehat{M}, \omega)\right|$. It also follows that if $X$ is a non-trivial, full strip involved in $K \in \mathcal{K}$, then $X^{\alpha}$ is a non-trivial full strip involved in $K^{\alpha} \in \widehat{\mathcal{K}}$. Finally, suppose that $\mathcal{F}_{1}(\mathcal{E}, M, \omega)=$ $\{A, B\}$ and $\mathcal{F}_{1}(\mathcal{E}, \widehat{M}, \omega)=\{\widehat{A}, \widehat{B}\}$. Equation (7) implies that we may assume without loss of generality that $\widehat{A}=A^{\alpha}$ and $\widehat{B}=B^{\alpha}$. Hence subgroups $A$ and $B$ are conjugate under an element $g \in G_{\omega}$, if and only if $\widehat{A}$ and $\widehat{B}$ are conjugate under $g^{\alpha}$, and, since $g \in G_{\omega}$ and $\alpha \in\left(\mathbb{N}_{\text {Sym } \Omega}(G)\right)_{\omega}$, we have $g^{\alpha} \in G_{\omega}$. This shows that the definitions of the classes $\mathrm{CD}_{1}(G), \mathrm{CD}_{\mathrm{S}}(G), \mathrm{CD}_{1 \mathrm{~S}}(G), \mathrm{CD}_{2 \sim}(G), \mathrm{CD}_{2 \varkappa}(G)$, and $\mathrm{CD}_{3}(G)$ do not depend on the choice of the plinth.

It is easy to see that the above classes are pairwise disjoint, and Theorem 6.1 implies that each Cartesian decomposition of $\mathrm{CD}_{\mathrm{tr}}(G)$ belongs to at least one of the classes. If $k=1$ and $M$ is a simple group, then the map $\sigma_{1}$ is the identity map $M \rightarrow M$, and so $\mathcal{K}_{\omega}(\mathcal{E})=\mathcal{F}_{1}(\mathcal{E}, M, \omega)$. Theorem 6.1 of [BPS04 implies that $|\mathcal{E}|=2$ and hence that $\left|\mathcal{F}_{1}(\mathcal{E}, M, \omega)\right|=2$. As $\mathcal{E} \in \mathrm{CD}_{\text {tr }}(G)$ we have that $G_{\omega}$ is transitive on $\mathcal{K}_{\omega}(\mathcal{E})$, and hence on $\mathcal{F}_{1}(\mathcal{E}, M, \omega)$. Thus $\mathcal{E} \in \mathrm{CD}_{2 \sim}(G)$. 
The examples given earlier in this paper show that for each $\mathrm{x} \in\{1, \mathrm{~S}, 1 \mathrm{~S}, 2 \sim$, $2 \nsim, 3\}$ there exists a group $G$ such that the class $\operatorname{CD}_{\mathrm{x}}(G)$ is non-empty. Considering the corresponding Cartesian systems, one can easily see that the Cartesian decomposition in the first example of the Introduction (given after Corollary 1.3) is in $\mathrm{CD}_{1}(T$ wr $K)$, the decomposition of the second is in $\mathrm{CD}_{2 \sim}\left(\left(\right.\right.$ Aut $\left.\mathrm{A}_{6}\right)$ wr $\left.K\right)$. The Cartesian decomposition in Example 2.1 belongs to $\mathrm{CD}_{2 \chi}(G)$, the one in Example 2.2 is in $\mathrm{CD}_{3}(G)$, and the one in Example 2.3 belongs to $\mathrm{CD}_{1 \mathrm{~S}}(G)$. Finally the decomposition in the example given for Theorem 1.2 in the Introduction is in $\mathrm{CD}_{\mathrm{S}}(K \mathrm{wr} Q)$. On the other hand no group $G$ exists such that each of the classes $\mathrm{CD}_{\mathrm{x}}(G)$ is non-empty. Indeed, it will follow from Theorems 6.3 and 7.3 that $\mathrm{CD}_{\mathrm{S}}(G) \neq \emptyset$ implies $\mathrm{CD}_{1}(G) \cup \mathrm{CD}_{1 \mathrm{~S}}(G) \cup \mathrm{CD}_{2 \sim}(G) \cup \mathrm{CD}_{2 \nsim}(G) \cup \mathrm{CD}_{3}(G)=\emptyset$, and $\mathrm{CD}_{1}(G) \cup \mathrm{CD}_{1 \mathrm{~S}}(G) \cup \mathrm{CD}_{2 \sim}(G) \cup \mathrm{CD}_{2 \nsim}(G) \cup \mathrm{CD}_{3}(G) \neq \emptyset$ implies $\mathrm{CD}_{\mathrm{S}}(G)=\emptyset$, for all $G$.

If an observant reader compares [BPS04, Theorem 6.1] to our 6-Class Theorem, then she finds that the Cartesian decompositions in [BPS04, Theorem 6.1(ii)] resemble the ones in $\mathrm{CD}_{3}(G)$. These Cartesian decompositions are, however, $G_{\omega^{-}}$ intransitive. This shows that allowing the plinth $M$ to be non-simple leads to a very rich theory, and it would not be realistic to expect results as explicit as the ones in BPS04. Nevertheless, it is both possible and desirable to give further details of the Cartesian decompositions in each class. The Cartesian decompositions in $\mathrm{CD}_{3}(G)$ were already described in $\mathrm{PS03}$. In this paper we describe classes $\mathrm{CD}_{1}(G)$ and $\mathrm{CD}_{\mathrm{S}}(G)$. Besides the fact that these classes are, in a sense, the easiest, their description also leads to the general result in Theorem 1.2. Thus we felt that their description belongs in this article. The classes $\mathrm{CD}_{1 \mathrm{~S}}(G), \mathrm{CD}_{2 \sim}(G)$, and $\mathrm{CD}_{2 \varkappa}(G)$ pose considerably more challenge, and they are addressed in a separate paper [PS].

Theorem 6.1 shows that the existence of a $G$-invariant Cartesian decomposition in a particular class may pose severe restriction on the structure of $G$. This is made more explicit in the following theorem. Recall that an innately transitive group $G$ with plinth $M$ has compound diagonal type if a point stabiliser $M_{\omega}$ is a subdirect subgroup of $M$ and is not simple.

Theorem 6.3. Suppose that $G$ is an innately transitive permutation group with a non-abelian plinth $M$, and let $T$ be the isomorphism type of a simple direct factor of $M$. Then the following all hold.

(a) If $\mathrm{CD}_{\mathrm{S}}(G) \neq \emptyset$, then $G$ is a quasiprimitive group with compound diagonal type.

(b) If $\mathrm{CD}_{1 \mathrm{~S}}(G) \cup \mathrm{CD}_{2 \sim}(G) \neq \emptyset$, then $T$ admits a factorisation with two proper, isomorphic subgroups, and hence $T$ is isomorphic to one of the groups $\mathrm{A}_{6}$, $\mathrm{M}_{12}, \mathrm{P} \Omega_{8}^{+}(q)$, or $\mathrm{Sp}_{4}\left(2^{a}\right)$ with $a \geqslant 2$.

(c) If $\mathrm{CD}_{2 \varkappa}(G) \neq \emptyset$, then $T$ admits a factorisation with proper subgroups.

(d) If $\mathrm{CD}_{3}(G) \neq \emptyset$, then $T$ admits a strong multiple factorisation, and hence $T$ is isomorphic to one of the groups $\mathrm{Sp}_{4 a}(2)$ with $a \geqslant 2, \mathrm{P} \Omega_{8}^{+}(3)$, or $\mathrm{Sp}_{6}(2)$.

Moreover, for each $\mathrm{x} \in\{\mathrm{S}, 1,1 \mathrm{~S}, 2 \sim, 2 \not, 3\}$ there is some $G$ as above such that $\mathrm{CD}_{\mathrm{x}}(G) \neq \emptyset$.

Proof. (a) Suppose now that $\mathrm{CD}_{\mathrm{S}}(G) \neq \emptyset$, and let $\mathcal{E} \in \mathrm{CD}_{\mathrm{S}}(G)$. Then the elements of $\mathcal{K}_{\omega}(\mathcal{E})$ are proper subdirect subgroups of $M$. By Theorem 6.1, the set $\mathcal{P}$ of supports $\operatorname{Supp} X$ of non-trivial strips $X$ involved in $\mathcal{K}_{\omega}(\mathcal{E})$ is non-empty and, by Theorem 6.1(d), $\mathcal{P}$ is a $G_{\omega}$-invariant partition of $\left\{T_{1}, \ldots, T_{k}\right\}$. Hence each $K \in$ 
$\mathcal{K}_{\omega}(\mathcal{E})$ is a direct product of those non-trivial strips that are involved in $K$, and those of the $T_{i}$ that are not covered by these strips. Therefore

$$
M_{\omega}=\bigcap_{K \in \mathcal{K}_{\omega}(\mathcal{E})} K=\prod_{X \text { is a nontrivial strip involved in } \mathcal{K}_{\omega}(\mathcal{E})} X .
$$

Thus $M_{\omega}$ is a subdirect subgroup of $M$, and hence $G$ has diagonal type. It follows from [BamP04, Proposition 5.5] that $G$ is quasiprimitive. Since there are at least $\left|\mathcal{K}_{\omega}(\mathcal{E})\right| \geqslant 2$ strips $X$ involved in $\mathcal{K}_{\omega}(\mathcal{E})$, we have that $M_{\omega} \nsucceq T$; therefore $G$ is of compound diagonal type.

(b)-(d) It follows from Theorem 6.1 and the definitions of $\mathrm{CD}_{2 \sim}(G)$ and $\mathrm{CD}_{2 \nsim}(G)$ that the claimed factorisations are admitted by $T$. Factorisations of finite simple groups with isomorphic subgroups were listed in [BPS04, Lemma 5.2], while strong multiple factorisation of finite simple groups were classified in [BadP98, Table V]. These results imply that the isomorphism type of $T$ in parts (b) and (d) is as claimed.

The final assertion follows from the discussion between Theorems 6.2 and 6.3 .

There are finite simple groups that admit no factorisations with proper subgroups, for example, $\mathrm{PSU}_{2 m+1}(q)$ (with a finite number of exceptions) and some sporadic groups (see the tables in [LPS90]). Therefore Theorem[6.3(c) also restricts the isomorphism type of $T$.

\section{The Cartesian decompositions in $\mathrm{CD}_{\mathrm{S}}(G)$ And in $\mathrm{CD}_{1}(G)$}

In this section we describe the elements of $\mathrm{CD}_{\mathrm{S}}(G)$ and $\mathrm{CD}_{1}(G)$ for an innately transitive permutation group $G$. First we show how to construct such Cartesian decompositions. Theorem 7.2 implies that our construction is as general as possible.

Example 7.1. Suppose that $G$ is an innately transitive permutation group with a non-abelian plinth $M$, and let $T_{1}, \ldots, T_{k}$ be the simple normal subgroups of $M$. Note that the conjugation action of $G$ permutes the set $\left\{T_{1}, \ldots, T_{k}\right\}$ transitively. Suppose that $\omega \in \Omega$ and $\mathcal{A}=\left\{A_{1}, \ldots, A_{\ell}\right\}$ is a $G$-invariant partition of $\left\{T_{1}, \ldots, T_{k}\right\}$ such that

$$
M_{\omega}=\sigma_{A_{1}}\left(M_{\omega}\right) \times \cdots \times \sigma_{A_{\ell}}\left(M_{\omega}\right) .
$$

Then for $i=1, \ldots, \ell$ let

$$
K_{i}=\sigma_{A_{i}}\left(M_{\omega}\right) \times \prod_{T_{j} \notin A_{i}} T_{j}
$$

and set $\mathcal{K}=\left\{K_{1}, \ldots, K_{\ell}\right\}$.

If $g \in G_{\omega}$ and $i \in\{1, \ldots, \ell\}$, then, since $M_{\omega}$ is normalised by $G_{\omega}$,

$$
\left(\sigma_{A_{i}}\left(M_{\omega}\right)\right)^{g}=\sigma_{A_{i}^{g}}\left(M_{\omega}^{g}\right)=\sigma_{A_{i}^{g}}\left(M_{\omega}\right) .
$$

Therefore

$$
K_{i}^{g}=\left(\sigma_{A_{i}}\left(M_{\omega}\right) \times \prod_{T_{j} \notin A_{i}} T_{j}\right)^{g}=\sigma_{A_{i}^{g}}\left(M_{\omega}\right) \times \prod_{T_{j} \notin A_{i}^{g}} T_{j},
$$

and so $K_{i}^{g} \in \mathcal{K}$. Hence $\mathcal{K}$ is $G_{\omega}$-invariant. It is also easy to see that equations (3) and (4) hold for $\mathcal{K}$, and hence $\mathcal{K}$ is a Cartesian system for $M$ with respect to $\omega$. It follows from the last displayed equation that the $G_{\omega}$-actions on $\mathcal{K}$ and on $\mathcal{A}$ are equivalent. Hence $G_{\omega}$ is transitive on $\mathcal{K}$, and so $\mathcal{E}(\mathcal{K}) \in \mathrm{CD}_{\text {tr }}(G)$. Moreover $\mathcal{E}(\mathcal{K}) \in \mathrm{CD}_{\mathrm{S}}(G)$ if $M_{\omega}$ is a subdirect subgroup of $M$, and $\mathcal{E}(\mathcal{K}) \in \mathrm{CD}_{1}(G)$ otherwise. 
Theorem 7.2. Let $G$ be an innately transitive group on $\Omega$ with a non-abelian plinth $M$, and let $\omega \in \Omega$. Let $\mathrm{x}=\mathrm{S}$ if $M_{\omega}$ is a subdirect subgroup of $M$, and let $\mathrm{x}=1$ otherwise. Then there is a bijection between the set $\operatorname{CD}_{\mathrm{x}}(G)$ and the set of $G$-invariant partitions $\left\{P_{1}, \ldots, P_{\ell}\right\}$ of $\left\{T_{1}, \ldots, T_{k}\right\}$ satisfying $M_{\omega}=\sigma_{P_{1}}\left(M_{\omega}\right) \times$ $\cdots \times \sigma_{P_{\ell}}\left(M_{\omega}\right)$, and each $\mathcal{E} \in \mathrm{CD}_{\mathrm{x}}(G)$ arises as in Example 7.1 .

Proof. If $\mathcal{P}=\left\{P_{1}, \ldots, P_{\ell}\right\}$ is a $G$-invariant partition of $\left\{T_{1}, \ldots, T_{k}\right\}$ such that

$$
M_{\omega}=\sigma_{P_{1}}\left(M_{\omega}\right) \times \cdots \times \sigma_{P_{\ell}}\left(M_{\omega}\right),
$$

then let $\mathcal{K}(\mathcal{P})=\left\{K_{1}, \ldots, K_{\ell}\right\}$ where, as in Example 7.1.

$$
K_{i}=\sigma_{P_{i}}\left(M_{\omega}\right) \times \prod_{T_{j} \notin P_{i}} T_{j} \quad \text { for all } i \in\{1, \ldots, \ell\} .
$$

Then, arguing as in Example 7.1, $\mathcal{K}(\mathcal{P})$ is a $G_{\omega}$-invariant Cartesian system of subgroups for $M$. It is easy to check that the map $\mathcal{P} \mapsto \mathcal{K}(\mathcal{P})$ is injective. If $M_{\omega}$ is not a subdirect subgroup of $M$, then $\mathcal{E}(\mathcal{K}(\mathcal{P})) \in \mathrm{CD}_{1}(G)$, and $\mathcal{E}(\mathcal{K}(\mathcal{P})) \in \mathrm{CD}_{\mathrm{S}}(G)$ otherwise. Hence we have an injective map $\mathcal{P} \mapsto \mathcal{E}(\mathcal{K}(\mathcal{P}))$ from the set of $G$ invariant partitions of $\left\{T_{1}, \ldots, T_{k}\right\}$ to $\mathrm{CD}_{\mathrm{x}}(G)$.

Now let $\mathcal{E} \in \mathrm{CD}_{1}(G)$ and set $\mathcal{K}_{\omega}(\mathcal{E})=\left\{K_{1}, \ldots, K_{\ell}\right\}$ and $\mathcal{P}=\left\{\left\{T_{i} \mid \sigma_{i}\left(K_{j}\right) \neq\right.\right.$ $\left.\left.T_{i}\right\} \mid j=1, \ldots, \ell\right\}$. Since $\mathcal{E} \in \mathrm{CD}_{1}(G)$ we have $\left|\mathcal{F}_{i}(\mathcal{E}, M, \omega)\right|=1$ for all $i$, that is, there is a unique $j$ such that $\sigma_{i}\left(K_{j}\right)<T_{i}$. Thus $\mathcal{P}$ is a partition of $\left\{T_{1}, \ldots, T_{k}\right\}$. As $\mathcal{E} \in \mathrm{CD}_{1}(G)$, it follows from Lemma 4.2 that if $i \in\{1, \ldots, k\}$ and $j \in\{1, \ldots, \ell\}$ such that $\sigma_{i}\left(K_{j}\right)=T_{i}$, then $T_{i} \leqslant K_{j}$. This implies that $\mathcal{K}_{\omega}(\mathcal{E})=\mathcal{K}(\mathcal{P})$, and we have $\mathcal{E}=\mathcal{E}(\mathcal{K}(\mathcal{P}))$. Thus in this case $\mathcal{P} \mapsto \mathcal{E}(\mathcal{K}(\mathcal{P}))$ is surjective, and so it is a bijection. On the other hand, if $M_{\omega}$ is a subdirect subgroup of $M$ and $\mathcal{E} \in \mathrm{CD}_{\mathrm{S}}(G)$, then Theorem 6.1 shows that $\mathcal{P}=\left\{\left\{T_{i} \mid T_{i} \nless K_{j}\right\} \mid j=1, \ldots, \ell\right\}$ is a partition of $\left\{T_{1}, \ldots, T_{k}\right\}$. It follows from the definition of $\mathcal{P}$ that $\mathcal{K}_{\omega}(\mathcal{E})=\mathcal{K}(\mathcal{P})$, and hence again $\mathcal{E}=\mathcal{E}(\mathcal{K}(\mathcal{P}))$, and the map $\mathcal{P} \mapsto \mathcal{E}(\mathcal{K}(\mathcal{P}))$ is a bijection in this case also.

Using the above characterisation of Cartesian decompositions, we can describe the class of Cartesian decompositions that are preserved by innately transitive groups with diagonal type, which, we recall, are defined as follows. Suppose that $G$ is an innately transitive permutation group on $\Omega$ with a non-abelian and non-simple plinth $M=T_{1} \times \cdots \times T_{k}$, where the $T_{i}$ are isomorphic to a non-abelian simple group $T$. As above, let $\sigma_{i}$ denote the $i$-th coordinate projection $M \rightarrow T_{i}$. Let $\omega \in \Omega$. Then $G$ has diagonal type if $\sigma_{i}\left(M_{\omega}\right)=T_{i}$ for some (and hence all) $i \in\{1, \ldots, k\}$. It follows from Scott's lemma that, in this case, $M_{\omega}$ is isomorphic to $T^{m}$ for some $m \leqslant k$. By [BamP04, Proposition 5.5], $M$ is the unique minimal normal subgroup of $G$, and so $G$ is quasiprimitive. We say that such a $G$ has simple diagonal type if $M_{\omega} \cong T$, and compound diagonal type otherwise (see also [BadP03]).

Theorem 7.3. Let $G$ be an innately transitive permutation group of diagonal type. Then $\mathrm{CD}(G) \neq \emptyset$ if and only if $G$ is a quasiprimitive group with compound diagonal type. Moreover, if $G$ is of compound diagonal type, then $\operatorname{CD}(G)=\operatorname{CD}_{\mathrm{S}}(G) \neq \emptyset$.

Proof. Let $M$ be the unique minimal normal subgroup of $G$. Let $T_{1}, \ldots, T_{k}$ be the simple normal subgroups of $M$, and let $T$ denote their common isomorphism type. Suppose that $M_{\omega}$ is a subdirect subgroup of $M$, and let $\mathcal{E}$ be an element in $\mathrm{CD}(G)$. It follows from BamP04, Proposition 5.5] that $G$ is a quasiprimitive group. For each $K \in \mathcal{K}_{\omega}(\mathcal{E})$ we have $M_{\omega} \leqslant K$, and so all elements of $\mathcal{K}_{\omega}(\mathcal{E})$ are subdirect subgroups of $M$. Let $K_{1}, K_{2} \in \mathcal{K}_{\omega}(\mathcal{E})$ be two distinct subgroups. Then 
$K_{1}, K_{2} \neq M$, and so, by Lemma 4.1, $K_{1}, K_{2}$ involve non-trivial full strips $X_{1}$ and $X_{2}$, say, and, by Theorem [5.1, $X_{1} \neq X_{2}$. Suppose that $T_{i_{1}} \in \operatorname{Supp} X_{1}$ and $T_{i_{2}} \in \operatorname{Supp} X_{2}$. Then $T_{i_{1}}^{g}=T_{i_{2}}$ for some $g \in G_{\omega}$, and so Theorem 5.1 implies that $X_{1}^{g}=X_{2}$. This, in turn, yields $K_{1}^{g}=K_{2}$. Thus $\mathcal{E} \in \mathrm{CD}_{\operatorname{tr}}(G)$, and then clearly $\mathcal{E} \in \mathrm{CD}_{\mathrm{S}}(G)$. Hence $\mathrm{CD}(G)=\mathrm{CD}_{\mathrm{S}}(G)$. Note that our argument also implies that if a non-trivial, full strip $X$ is involved in $\mathcal{K}$, then it is also involved in $M_{\omega}$. The supports of the full strips involved in $M_{\omega}$ form a $G$-invariant partition $\mathcal{P}$ of $\left\{T_{1}, \ldots, T_{k}\right\}$ such that $M_{\omega}=\prod_{P \in \mathcal{P}} \sigma_{P}\left(M_{\omega}\right)$. Since $|\mathcal{P}| \geqslant 2, M_{\omega}$ is not simple, and so $G$ has compound diagonal type. Conversely, if $G$ has compound diagonal type, then, by Theorem $7.2, \mathrm{CD}(G)$ is non-empty, and we showed above that, in this case, $\mathrm{CD}(G)=\mathrm{CD}_{\mathrm{S}}(G)$.

\section{INCLUSIONS IN WREATH PRODUCTS}

This section is devoted to proving Theorems 1.1, 1.2 and Corollary 1.3. Throughout the section we assume that the common hypotheses of Theorems 1.1 and 1.2 hold. Thus, let $H$ be a quasiprimitive, almost simple permutation group acting on a set $\Gamma$, and let $U$ denote its simple normal subgroup. Set $W=H \mathrm{wr} \mathrm{S}_{\ell} \cong$ $H^{\ell} \rtimes \mathrm{S}_{\ell}$, and consider $W$ as a permutation group on $\Gamma^{\ell}$ in product action. Let $N=U_{1} \times \cdots \times U_{\ell} \cong U^{\ell}$ denote the unique minimal normal subgroup of $W$. As there is a natural isomorphism $U \rightarrow U_{i}$ for each $i \in\{1, \ldots, \ell\}$, we consider the $U_{i}$ as subgroups of Sym $\Gamma$. Let $G$ be a finite, innately transitive permutation group acting on $\Gamma^{\ell}$ with a non-abelian plinth $M=T_{1} \times \cdots \times T_{k}$, where $T_{1}, \ldots, T_{k}$ are finite simple groups all isomorphic to a group $T$. Assume that $G \leqslant W$. Note that at this stage we do not assume that $G$ projects onto a transitive subgroup of $\mathrm{S}_{\ell}$.

Let us introduce some extra notation to facilitate our investigation of the situations described by Theorems 1.1 and 1.2. As before, $\sigma_{i}$ denotes the natural projection $M \rightarrow T_{i}$, and, for $i=1, \ldots, \ell$, let $\mu_{i}$ denote the natural projection $N \rightarrow U_{i}$. Note that $W$ preserves the natural Cartesian decomposition $\mathcal{E}=\left\{\Gamma_{1}, \ldots, \Gamma_{\ell}\right\}$ of $\Gamma^{\ell}$, where

$$
\Gamma_{i}=\left\{\left\{\left(\gamma_{1}, \ldots, \gamma_{\ell}\right) \mid \gamma_{i}=\gamma\right\} \mid \gamma \in \Gamma\right\} \quad \text { for } \quad i \in\{1, \ldots, \ell\} .
$$

Fix $\gamma \in \Gamma$ and let $\omega=(\gamma, \ldots, \gamma)$. Let $\mathcal{K}=\left\{\mathcal{K}_{1}, \ldots, K_{\ell}\right\}$ be the $G_{\omega}$-invariant Cartesian system in $M$ with respect to $\omega$ corresponding to $\mathcal{E}$. It follows from the definition of the product action that the $W$-actions on $\mathcal{E}$ and on the set of simple direct factors $U_{1}, \ldots, U_{\ell}$ of $N$ are equivalent.

The proof of the next lemma uses the Schreier Conjecture that the outer automorphism group of a finite simple group is soluble. The validity of the Schreier Conjecture follows from the finite simple group classification.

Lemma 8.1. We have that $M \leqslant N$.

Proof. Let $B$ denote the base group $H^{\ell}$ of $W$. It follows from the definition of the product action that the pointwise stabiliser in $W$ of $\mathcal{E}$ coincides with $B$, and so [BPS04, Proposition 2.1] implies that $M \leqslant B$. Now, as $M$ is a minimal normal subgroup of $G$, we have that either $M \leqslant N$ or $M \cap N=1$. If $M \leqslant N$, then we are done, so assume that $M \cap N=1$. Then $(M N) / N \cong M /(M \cap N) \cong M$, and so $M$ can be viewed as a subgroup of $B / N$. On the other hand, $B / N \cong(H / U)^{\ell}$. By the Schreier Conjecture, $H / U$ is a soluble group, and therefore so is $B / N$. Hence assuming that $M \cap N=1$ leads to the incorrect conclusion that $M$ is a soluble group. Therefore $M \leqslant N$ must hold. 
Note that $M$ is a characteristically simple group and each of its normal subgroups is a product of some of the $T_{i}$. If $L$ is a normal subgroup of $M$, then the quotient $M / L$ can naturally be identified with the subgroup $\prod_{T_{i} \nless L} T_{i}$, and in the future this identification will be used without further comment. For a subgroup $K<M$, Core $_{M}(K)=\bigcap_{m \in M} K^{m}$ is the largest normal subgroup of $M$ contained in $K$.

Lemma 8.2. Let $i \in\{1, \ldots, \ell\}$. Then

$$
M^{\Gamma_{i}}=\prod_{T_{j} \nless K_{i}} T_{j} \cong T^{s_{i}}
$$

for some $s_{i} \geqslant 1$, and $\mu_{i}(M)$ is permutationally isomorphic to $M^{\Gamma_{i}}$. Moreover, $\mu_{i}(M)$ is a transitive subgroup of $U_{i}$, and if $s_{i} \geqslant 2$, then $U=$ Alt $\Gamma$.

Proof. Note that $K_{i}$ is the stabiliser of a point for the $M$-action on $\Gamma_{i}$, and the kernel of this action is Core ${ }_{M}\left(K_{i}\right)$. Now $T_{j} \leqslant \operatorname{Core}_{M}\left(K_{i}\right)$ if and only if $T_{j} \leqslant K_{i}$, and so (8) holds.

Let $\alpha$ denote the bijection $\Gamma \rightarrow \Gamma_{i}$ mapping

$$
\gamma \mapsto\left\{\left(\gamma_{1}, \ldots, \gamma_{\ell}\right) \mid \gamma_{i}=\gamma\right\} \quad \text { for all } \gamma \in \Gamma .
$$

The map $\mu_{i}$ can be considered as a permutation representation of $M$ in Sym $\Gamma$. We claim that the $M$-actions on $\Gamma$ and $\Gamma_{i}$ are equivalent via the bijection $\alpha$. Let $\gamma \in \Gamma$ and $m \in M$. Then

$$
\alpha(\gamma m)=\left\{\left(\gamma_{1}, \ldots, \gamma_{\ell}\right) \mid \gamma_{i}=\gamma m\right\}=\left\{\left(\gamma_{1}, \ldots, \gamma_{\ell}\right) \mid \gamma_{i}=\gamma\right\} m=\alpha(\gamma) m .
$$

Therefore our claim holds. As $\Gamma_{i}$ is an $M$-invariant partition of $\Omega$, the group $M$ is transitive on $\Gamma_{i}$, and so $\mu_{i}(M)$ is transitive on $\Gamma$. Thus the factorisation $\mu_{i}(M)\left(U_{i}\right)_{\gamma}=U_{i}$ holds. Also note that $\mu_{i}(M)$ is a homomorphic image of $M$, and so $\mu_{i}(M) \cong T^{s_{i}}$ for some $s_{i}$. Let $I$ denote the set of indices $i$ such that $s_{i} \geqslant 1$. Then $M \leqslant \prod_{i \in I} U_{i}$, and so $\prod_{i \in I} U_{i}$ is a transitive normal subgroup of $N$. However, by the definition of the product action, $\prod_{j \in J} U_{j}$ is intransitive if $J$ is a proper subset of $\{1, \ldots, \ell\}$. Hence $I=\{1, \ldots, \ell\}$, and so $s_{i} \geqslant 1$ for all $i$. If $s_{i} \geqslant 2$ for some $i$, then BadP03, Theorem 1.4] implies that $U=$ Alt $\Gamma$.

These results enable us to show that, for four of the six classes identified by the 6-Class Theorem, the corresponding embedding of $G$ belongs to Theorem 1.1(c).

Lemma 8.3. Suppose that $\mathcal{E} \in \mathrm{CD}_{\operatorname{tr}}(G)$. If $\mu_{i}(M) \cong T^{s_{i}}$ with $s_{i} \geqslant 2$ for some $i \in\{1, \ldots, \ell\}$, then Theorem 1.1 (c) is valid.

Proof. Suppose that $\mu_{i}(M) \cong T^{s_{i}}$ and $s_{i} \geqslant 2$ for some $i \geqslant 1$. It follows from Lemma 8.2 that in this case $U=$ Alt $\Gamma$. Also, note that there are exactly $s_{i}$ indices $j$ such that $\mu_{i}\left(T_{j}\right) \cong T$. On the other hand, in cases (a) and (b) for each $i \in\{1, \ldots, \ell\}$ there is a unique $j \in\{1, \ldots, k\}$ such that $\mu_{i}\left(T_{j}\right) \cong T$. Therefore Theorem 1.1(c) holds.

Lemma 8.4. (a) If $\mathcal{E} \in \mathrm{CD}_{\mathrm{S}}(G) \cup \mathrm{CD}_{1 \mathrm{~S}}(G) \cup \mathrm{CD}_{3}(G) \cup \mathrm{CD}_{2 \nsim}(G)$, then $\mu_{i}(M) \cong T^{s}$ with $s$ independent of $i$ and $s \geqslant 2$, and Theorem 1.1(c) is valid.

(b) If $\mathcal{E} \in \mathrm{CD}_{1}(G)$, then either case (a) or case (c) of Theorem 1.1 is valid.

(c) If $\mathcal{E} \in \mathrm{CD}_{2 \sim}(G)$, then either case (b) or case (c) of Theorem 1.1 is valid.

Proof. In each case, $G$ is transitive on $\mathcal{E}$, and $M$ is a normal subgroup of $G$, and so the permutation groups $M^{\Gamma_{i}}$ are pairwise permutationally isomorphic. Thus 
$M^{\Gamma_{i}} \cong T^{s}$ for some $s \geqslant 1$ independent of $i$, and, by Lemma $8.2, \mu_{i}(M) \cong T^{s}$. If $s \geqslant 2$, then Lemma 8.3 implies that case (c) of the theorem holds.

(a) Let $\mathcal{E} \in \mathrm{CD}_{\mathrm{S}}(G) \cup \mathrm{CD}_{1 \mathrm{~S}}(G) \cup \mathrm{CD}_{3}(G) \cup \mathrm{CD}_{2 \chi}(G)$. By the discussion in the previous paragraph, it suffices to verify that $\mu_{1}(M) \cong T^{s}$ with $s \geqslant 2$. Note that, by Lemma 8.2. $\mu_{1}(M)=\prod_{T_{j} \nless K_{1}} T_{j}$. If $\mathcal{E} \in \mathrm{CD}_{\mathrm{S}}(G) \cup \mathrm{CD}_{1 \mathrm{~S}}(G)$, then $K_{1}$ involves a non-trivial, full strip, and so $s \geqslant 2$ follows immediately.

Suppose next that $\mathcal{E} \in \mathrm{CD}_{3}(G)$. Then $\mathcal{F}_{1}(\mathcal{E}, M, \omega)=\{A, B, C\}$ for some subgroups $A, B$, and $C$ of $T_{1}$, such that $A, B$, and $C$ form a strong multiple factorisation of $T_{1}$. Hence [BadP98, Table V] yields that the subgroups $A, B$, and $C$ represent three distinct isomorphism types. Then there are pairwise distinct indices $j_{1}, j_{2}, j_{3} \in\{1, \ldots, \ell\}$ such that $\sigma_{1}\left(K_{j_{1}}\right)=A, \sigma_{1}\left(K_{j_{2}}\right)=B, \sigma_{1}\left(K_{j_{3}}\right)=C$. Let $g_{1}, g_{2}, g_{3} \in G_{\omega}$ such that $K_{j_{1}}^{g_{1}}=K_{j_{2}}^{g_{2}}=K_{j_{3}}^{g_{3}}=K_{1}$. Let $i_{1}, i_{2}, i_{3} \in\{1, \ldots, k\}$ such that $T_{1}^{g_{1}}=T_{i_{1}}, T_{1}^{g_{2}}=T_{i_{2}}, T_{1}^{g_{3}}=T_{i_{3}}$. Then $\sigma_{i_{1}}\left(K_{1}\right)=\sigma_{1}\left(K_{j_{1}}\right)^{g_{1}}=A^{g_{1}}$, $\sigma_{i_{2}}\left(K_{1}\right)=\sigma_{1}\left(K_{j_{2}}\right)^{g_{2}}=B^{g_{2}}$, and $\sigma_{i_{3}}\left(K_{1}\right)=\sigma_{1}\left(K_{j_{3}}\right)^{g_{3}}=C^{g_{3}}$. As $A$, B , and $C$ are pairwise non-isomorphic, it follows that $i_{1}, i_{2}, i_{3}$ are also pairwise distinct. Thus (8) implies that $\mu_{1}(M) \cong T^{s}$ for some $s \geqslant 3$.

Suppose finally that $\mathcal{E} \in \mathrm{CD}_{2 \nsim}(G)$ such that $\mathcal{F}_{1}(\mathcal{E}, M, \omega)=\{A, B\}$, where $A, B \leqslant T_{1}$ are not conjugate under $G_{\omega}$. Then it follows that there are indices $j_{1}, j_{2} \in\{1, \ldots, \ell\}$ such that $\sigma_{1}\left(K_{j_{1}}\right)=A$ and $\sigma_{1}\left(K_{j_{2}}\right)=B$. Let $g_{1}, g_{2} \in G_{\omega}$ be such that $K_{j_{1}}^{g_{1}}=K_{j_{2}}^{g_{2}}=K_{1}$. Then $T_{1}^{g_{1}}=T_{i_{1}}$ and $T_{1}^{g_{2}}=T_{i_{2}}$ for some $i_{1}, i_{2} \in$ $\{1, \ldots, k\}$. Hence $\sigma_{i_{1}}\left(K_{1}\right)=\sigma_{1}\left(K_{j_{1}}\right)^{g_{1}}=A^{g_{1}}$ and $\sigma_{i_{2}}\left(K_{1}\right)=\sigma_{1}\left(K_{j_{2}}\right)^{g_{2}}=B^{g_{2}}$. As $A$ and $B$ are not conjugate under $G_{\omega}$, we have that $A^{g_{1}} \neq B^{g_{2}}$, and so $i_{1} \neq i_{2}$. Thus (8) implies that $\mu_{i}(M) \cong T^{s}$ where $s \geqslant 2$, and the result follows.

(b) As noted above, if $\mu_{i}(M) \cong T^{s}$ with $s \geqslant 2$, then Theorem 1.1(c) holds. Assume now that $\mu_{i}(M) \cong T$. As $\mathcal{E} \in \mathrm{CD}_{1}(G)$, for all $i \in\{1, \ldots, k\}$ there is a unique $j \in\{1, \ldots, \ell\}$ such that $T_{i} \nless K_{j}$. This means that for all $i \in\{1, \ldots, k\}$ there is a unique $j \in\{1, \ldots, \ell\}$ such that $\mu_{j}\left(T_{i}\right) \neq 1$, and so $T_{i} \leqslant U_{j}$. On the other hand, as $\mu_{i}(M) \cong T$, for all $j \in\{1, \ldots, \ell\}$ there is a unique $i \in\{1, \ldots, k\}$ such that $T_{i} \leqslant U_{j}$. Therefore $\ell=k$ and the $T_{i}$ and the $U_{j}$ can be indexed so that $T_{1} \leqslant U_{1}, \ldots, T_{k} \leqslant U_{k}$, and so Theorem 1.1(a) holds.

(c) As in part (b), either Theorem 1.1 (c) holds or $\mu_{i}(M) \cong T$ for all $i$; assume the latter. As $\mathcal{E} \in \mathrm{CD}_{2 \sim}(G)$, for all $i \in\{1, \ldots, k\}$, there are exactly two indices $j \in\{1, \ldots, \ell\}$ such that $\mu_{j}\left(T_{i}\right) \neq 1$. If $j_{1}, j_{2} \in\{1, \ldots, \ell\}$ are these indices, then we obtain that $T_{i} \leqslant U_{j_{1}} \times U_{j_{2}}$. On the other hand, as $\mu_{j}(M) \cong T$, for all $j \in\{1, \ldots, \ell\}$ there is a unique $i \in\{1, \ldots, k\}$ such that $\mu_{j}\left(T_{i}\right) \neq 1$. Counting the pairs in the set

$$
\left\{(i, j) \mid i \in\{1, \ldots, k\}, j \in\{1, \ldots, \ell\}, T_{i} \nless K_{j}\right\}
$$

we obtain that $\ell=2 k$ and the $T_{i}$ and the $U_{i}$ can be indexed so that

$$
T_{1} \leqslant U_{1} \times U_{2}, T_{2} \leqslant U_{3} \times U_{4}, \ldots, T_{k} \leqslant U_{2 k-1} \times U_{2 k} .
$$

Note that $U_{1} \times U_{2}$ can be viewed as a permutation group acting on $\Gamma_{1} \times \Gamma_{2}$ preserving the natural Cartesian decomposition formed by the $\left(U_{1} \times U_{2}\right)$-invariant partitions $\Gamma_{1}$ and $\Gamma_{2}$. Choose $\gamma_{1} \in \Gamma_{1}, \gamma_{2} \in \Gamma_{2}$ and set $\omega=\left(\gamma_{1}, \gamma_{2}\right)$. Then [BPS04, Theorem 6.1] implies that the isomorphism type $T$ of $T_{1}$, and the stabilisers $\left(T_{1}\right)_{\gamma_{1}}$, $\left(T_{1}\right)_{\gamma_{2}}$ and $\left(T_{1}\right)_{\omega}$ are as in Table 2. Hence $T_{1}$ acts primitively on both $\Gamma_{1}$ and $\Gamma_{2}$. Thus $\left(U_{1}\right)^{\Gamma_{1}}$ and $\left(U_{2}\right)^{\Gamma_{2}}$ are primitive permutation groups. Since $\left(T_{1}\right)^{\Gamma_{1}} \leqslant\left(U_{1}\right)^{\Gamma_{1}}$ and $\left(T_{2}\right)^{\Gamma_{2}} \leqslant\left(U_{2}\right)^{\Gamma_{2}}$, the results of [LPS87] yield that $U$ and $T$ are as in the corresponding columns of Table 1. Thus Theorem 1.1(b) holds. 
TABLE 2. The stabilisers in $T_{1}$

\begin{tabular}{|c|c|c|c|}
\hline & $T$ & $\left(T_{1}\right)_{\gamma_{1}},\left(T_{1}\right)_{\gamma_{2}}$ & $\left(T_{1}\right)_{\omega}$ \\
\hline \hline 1 & $\mathrm{~A}_{6}$ & $\mathrm{~A}_{5}, \tau\left(\mathrm{A}_{5}\right)\left(\tau \notin \mathrm{S}_{6}\right)$ & $\mathrm{D}_{10}$ \\
\hline 2 & $\mathrm{M}_{12}$ & $\mathrm{M}_{11}, \tau\left(\mathrm{M}_{11}\right)\left(\tau \notin \mathrm{M}_{12}\right)$ & $\mathrm{PSL}_{2}(11)$ \\
\hline 3 & $\mathrm{P} \Omega_{8}^{+}(q)$ & $\Omega_{7}(q), \tau\left(\Omega_{7}(q)\right)(\tau$ a triality $)$ & $\mathrm{G}_{2}(q)$ \\
\hline 4 & $\mathrm{Sp}_{4}\left(2^{a}\right), a \geqslant 2$ & $\mathrm{Sp}_{2}\left(2^{2 a}\right) \cdot 2, \mathrm{O}_{4}^{-}(q)$ & $\mathrm{D}_{q^{2}+1} \cdot 2$ \\
\hline
\end{tabular}

The proof of Theorem 1.1 is now very easy.

Proof of Theorem 1.1. The theorem follows from the 6-Class Theorem (Theorem 6.2) and Lemma 8.4.

Next we prove Theorem 1.2

Proof of Theorem 1.2. Let $G$ and $W$ be as in Theorem 1.2. Then $\operatorname{CD}(G) \neq \emptyset$, and hence, by Theorem $7.3, G$ is a quasiprimitive group with compound diagonal type and the Cartesian decomposition $\mathcal{E}$ corresponding to the product action of $W$ belongs to $\mathrm{CD}_{\mathrm{S}}(G)$. In particular, $\mathcal{E} \in \mathrm{CD}_{\mathrm{tr}}(G)$, and so $G$ projects onto a transitive subgroup of $\mathrm{S}_{\ell}$. Thus part (a) is proved. It follows from Lemma 8.4 that the inclusion $G \leqslant W$ is as in Theorem 1.1(c), and so $U=$ Alt $\Gamma$. Thus part (b) holds.

By Lemma 8.1, $M \leqslant N$. Let $\omega \in \Omega$ and consider the Cartesian system $\mathcal{K}_{\omega}(\mathcal{E})$ for $M$. If $K \in \mathcal{K}_{\omega}(\mathcal{E})$, then $M_{\omega} \leqslant K$, and so $K$ is a subdirect subgroup of $M$. Thus Lemma 4.1 implies that $K$ is the direct product of pairwise disjoint, full strips. By Theorem 5.1, if $X_{1}$ and $X_{2}$ are non-trivial, full strips involved in $\mathcal{K}_{\omega}(\mathcal{E})$, then $X_{1}$ and $X_{2}$ are disjoint. Thus if $T_{i} \nless K_{j}$ for some $i \in\{1, \ldots, k\}$ and $j \in\{1, \ldots, \ell\}$, then $T_{i} \leqslant K_{j^{\prime}}$ for all $j^{\prime} \in\{1, \ldots, \ell\} \backslash\{j\}$. Hence the $T_{1}, \ldots, T_{k}$ and the $U_{1}, \ldots, U_{\ell}$ can be indexed such that

$$
M^{\Gamma_{1}}=T_{1} \times \cdots \times T_{m}, \ldots, M^{\Gamma_{\ell}}=T_{(\ell-1) m+1}, \ldots, T_{\ell m} .
$$

Thus $k=\ell m$ and by Lemmas 8.2 and $8.4, m \geqslant 2$. Also

$$
T_{1} \times \cdots \times T_{m} \leqslant U_{1}, \ldots, T_{(\ell-1) m+1} \times \ldots \times T_{\ell m} \leqslant U_{\ell},
$$

and statement $(\mathrm{c})$ is also valid.

Finally we prove Corollary 1.3 ,

Proof of Corollary 1.3. Let $G$ be an innately transitive group on $\Omega$ with simple diagonal type, and let $M$ be the unique minimal normal subgroup of $G$, whose existence is guaranteed by [BamP04, Proposition 5.5]. Assume that the result is not true for $G$, so $G \leqslant W$ where $W$ is permutationally isomorphic to Sym $\Gamma$ wr $\mathrm{S}_{\ell}$ with some $|\Gamma| \geqslant 2$ and $\ell \geqslant 2$. Let $\mathcal{E}$ denote the natural Cartesian decomposition of $\Omega$ corresponding to the product action of $W$. Then [BPS04, Proposition 2.1] implies that $M$ lies in the pointwise stabiliser in $W$ of $\mathcal{E}$, and so $M$ is a subgroup of the base group $(\operatorname{Sym} \Gamma)^{\ell}$ of $W$. As $M$ is a non-abelian characteristically simple group and Sym $\Gamma$ is soluble for $|\Gamma| \leqslant 4$, we obtain that $|\Gamma| \geqslant 5$. Thus Theorem 1.2 implies that $G$ has compound diagonal type, which is a contradiction. 


\section{REFERENCES}

[BadP98] Robert W. Baddeley and Cheryl E. Praeger. On classifying all full factorisations and multiple-factorisations of the finite almost simple groups, J. Algebra, 204(1):129-187, 1998. MR1623953 (99h:20019)

[BadP03] R. W. Baddeley and C. E. Praeger. On primitive overgroups of quasiprimitive permutation groups, J. Algebra, 263(2):294-344, 2003. MR1978653 (2004b:20005)

[BPS04] Robert W. Baddeley, Cheryl E. Praeger and Csaba Schneider. Transitive simple subgroups of wreath products in product action, J. Austral. Math. Soc., 77(1):55-72, 2004. MR2069025

[BamP04] John Bamberg and Cheryl E. Praeger. Finite permutation groups with a transitive minimal normal subgroup, Proc. London. Math. Soc. 89(1):71-103, 2004. MR2063660

[Bau97] Barbara Baumeister. Factorizations of primitive permutation groups. J. Algebra, 194(2):631-653, 1997. MR1467170 (99i:20004)

[DM96] John D. Dixon and Brian Mortimer. Permutation groups, Springer-Verlag, New York, 1996. MR:1409812 (98m:20003)

[Kov89a] L. G. Kovács. Primitive subgroups of wreath products in product action. Proc. London Math. Soc. (3), 58(2):306-322, 1989. MR0977479 (90a:20008)

[Kov89b] L. G. Kovács. Wreath decompositions of finite permutation groups. Bull. Austral. Math. Soc., 40(2):255-279, 1989. MR1012834 (90j:20006)

[LPS87] Martin W. Liebeck, Cheryl E. Praeger, and Jan Saxl. A classification of the maximal subgroups of the finite alternating and symmetric groups, J. Algebra, 111(2):365-383, 1987. MR0916173 (89b:20008)

[LPS90] Martin W. Liebeck, Cheryl E. Praeger, and Jan Saxl. The maximal factorizations of the finite simple groups and their automorphism groups. Mem. Amer. Math. Soc., 86(432):iv+151, 1990. MR1016353 (90k:20048)

[Pra90] Cheryl E. Praeger. The inclusion problem for finite primitive permutation groups, Proc. London Math. Soc. (3), 60(1):68-88, 1990. MR1023805 (90j:20009)

[Pra93] Cheryl E. Praeger. An O'Nan-Scott theorem for finite quasiprimitive permutation groups and an application to 2-arc transitive graphs. J. London Math. Soc. (2), 47(2):227-239, 1993. MR1207945 (94f:05068)

[PS02] Cheryl E. Praeger and Csaba Schneider. Factorisations of characteristically simple groups. J. Algebra, 255(1):198-220, 2002. MR.1935043 (2003i:20046)

[PS03] Cheryl E. Praeger and Csaba Schneider. Ordered triple designs and wreath products of groups. In Darlene R. Goldstein (Ed.) Science and Statistics: A Festschrift for Terry Speed. Institute of Mathematical Statistics, Lecture Notes - Monograph Series, volume 40, pages 103-113, 2003. MR2004334

[PS] Cheryl E. Praeger and Csaba Schneider. Three types of inclusions of innately transitive permutation groups into wreath products in product action. Submitted arxiv.org/math.GR/0406600.

[Sco80] Leonard L. Scott. Representations in characteristic $p$. In The Santa Cruz Conference on Finite Groups (Univ. California, Santa Cruz, Calif., 1979), pages 319-331. Amer. Math. Soc., Providence, R.I., 1980. MR0604599 (82e:20052)

32 Arbury Road, Cambridge CB4 2JE, United Kingdom

E-mail address: robert.baddeley@ntworld.com

School of Mathematics and Statistics, The University of Western Australia, 35 Stirling Highway 6009 Crawley, Western Australia

E-mail address: praeger@maths.uwa.edu.au

$U R L$ : www . maths. uwa.edu.au/ p praeger

Informatics Laboratory, Computer and Automation Research Institute of the Hungarian Academy of Sciences, P.O. Box 63, 1518 Budapest, Hungary

E-mail address: csaba.schneider@sztaki.hu

$U R L$ : www.sztaki.hu/ schneider 